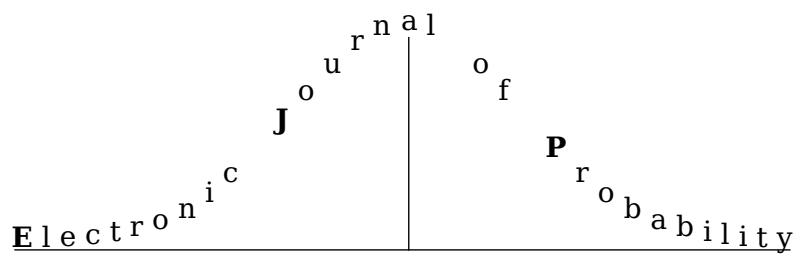

Electron. J. Probab. 26 (2021), article no. 12, 1-26.

ISSN: 1083-6489 https://doi.org/10.1214/20-EJP555

\title{
On absolute continuity and singularity of multidimensional diffusions
}

\author{
David Criens*
}

\begin{abstract}
Consider two laws $P$ and $Q$ of multidimensional possibly explosive diffusions with common diffusion coefficient $\mathfrak{a}$ and drift coefficients $\mathfrak{b}$ and $\mathfrak{b}+\mathfrak{a} \mathfrak{c}$, respectively, and the law $P^{\circ}$ of an auxiliary diffusion with diffusion coefficient $\langle\mathfrak{c}, \mathfrak{a c}\rangle^{-1} \mathfrak{a}$ and drift coefficient $\langle\mathfrak{c}, \mathfrak{a c}\rangle^{-1} \mathfrak{b}$. We show that $P \ll Q$ if and only if the auxiliary diffusion $P^{\circ}$ explodes almost surely and that $P \perp Q$ if and only if the auxiliary diffusion $P^{\circ}$ almost surely does not explode. As applications we derive a Khasminskii-type integral test for absolute continuity and singularity, an integral test for explosion of time-changed Brownian motion, and we discuss applications to mathematical finance.
\end{abstract}

Keywords: absolute continuity; singularity; multidimensional diffusion; uniformly integrable martingale; explosion; integral test; perpetual integral; random time change.

MSC2020 subject classifications: 60J60; 60G44; 60H10; 91B70.

Submitted to EJP on April 30, 2020, final version accepted on November 12, 2020.

\section{Introduction}

Consider two laws $P$ and $Q$ of multidimensional possibly explosive diffusions with common diffusion coefficient $\mathfrak{a}$ and drift coefficients $\mathfrak{b}$ and $\mathfrak{b}+\mathfrak{a c}$, respectively. We are interested in finding analytic conditions for the absolute continuity $P \ll Q$ and the singularity $P \perp Q$. Such conditions are of interest in many branches of probability theory. In mathematical finance, for instance, mutual absolute continuity is of importance in the study of the absence of arbitrage, see [8, 15].

For one-dimensional diffusions precise integral tests were proven in [9] under the Engelbert-Schmidt conditions. For multidimensional diffusions the situation is less well-understood and only a few analytic conditions are known, see [3] for an integral test for Fuchsian diffusions. Here, a diffusion is called Fuchsian if the coefficients $\mathfrak{a}$ and $\mathfrak{b}$ are locally Hölder continuous, $\mathfrak{a}$ is uniformly elliptic and $\sup _{x \in \mathbb{R}^{d}}\|\mathfrak{b}(x)\|(1+\|x\|)<\infty$.

*Technical University of Munich, Germany.

E-mail: david.criens@tum.de 
The starting point for our research is the following probabilistic characterization of absolute continuity and singularity: Let $X$ be the coordinate process and set

$$
A_{t} \triangleq \int_{0}^{t \wedge \theta}\left\langle\mathfrak{c}\left(X_{s}\right), \mathfrak{a}\left(X_{s}\right) \mathfrak{c}\left(X_{s}\right)\right\rangle d s, \quad t \in \mathbb{R}_{+},
$$

where $\theta$ is the explosion time. It has been proven in [3,12] that $P \ll Q$ is equivalent to $P\left(A_{\theta}<\infty\right)=1$ and that $P \perp Q$ is equivalent to $P\left(A_{\theta}=\infty\right)=1$. In other words, $P \ll Q$ and $P \perp Q$ are characterized by $P$-a.s. divergence and convergence of the perpetual integral $A_{\theta}$. Again, these properties are well-understood for one-dimensional diffusions, see $[13,23,28,32]$, and it seems that less work has been done for the multidimensional case, see [16, 42] for results concerning Bessel processes, [12] for some conditions in radial cases, and [24] for results on divergence in case $X$ is a conservative Feller process possibly with jumps.

In $[5,13,23,24]$ the perpetual integral $A_{\theta}$ was related to the hitting time of a timechanged process. We pick up this idea and prove the following: Let $\mathfrak{f}: \mathbb{R}^{d} \rightarrow(0, \infty)$ be a Borel function which is locally bounded away from zero and infinity. Under the assumptions that the diffusion $P$ exists and that $\mathfrak{b}$ and $\mathfrak{a}$ are locally bounded, we show existence of a diffusion $P^{\circ}$ with diffusion coefficient $\mathfrak{f}^{-1} \mathfrak{a}$ and drift coefficient $\mathfrak{f}^{-1} \mathfrak{b}$ such that the law of the perpetual integral

$$
T_{\theta} \triangleq \int_{0}^{\theta} \mathfrak{f}\left(X_{s}\right) d s
$$

under $P$ coincides with the law of the explosion time $\theta$ under $P^{\circ}$. Furthermore, we show that $P^{\circ}$ is unique whenever $P$ is unique.

Returning to our initial problem, we note that in case $\mathfrak{f}=\langle\mathfrak{c}, \mathfrak{a c}\rangle$ the absolute continuity $P \ll Q$ is equivalent to $P^{\circ}(\theta<\infty)=1$ and the singularity $P \perp Q$ is equivalent to $P^{\circ}(\theta=\infty)=1$. This observation is very useful, because the literature contains many conditions for explosion and non-explosion of multidimensional diffusions, see $[27,33,40]$. For illustration, we formulate a Khasminskii-type integral test for absolute continuity and singularity.

The result can also be applied in the converse direction: In case we have criteria for absolute continuity and singularity, these can be used to deduce explosion criteria for time-changed diffusions. To illustrate this, we derive an integral test for almost sure explosion and non-explosion of time-changed Brownian motion, using results on singularity of Fuchsian diffusions proven in [3].

The absolute continuity $P \ll Q$ is intrinsically connected to the uniform integrable (UI) $Q$-martingale property of a certain stochastic exponential (see Eq. (2.2) below), which has been studied for one-dimensional diffusions in [29]. Independent of the dimension, for the conservative case it is known that the loss of the martingale property has a one-to-one relation to the explosion of an auxiliary diffusion, see, e.g., [7, 39]. This turned out to be wrong in the non-conservative setting of [29]. Our result shows that for the UI martingale property the statement is true irrespective whether the diffusions are conservative or non-conservative.

As a third application, we use the relation of the UI martingale property and absolute continuity to study a problem in mathematical finance: More precisely, for certain exponential diffusion models with infinite time horizon we derive analytic criteria for the existence of an equivalent local martingale measure (ELMM), and explosion criteria for the existence of an equivalent martingale measure (EMM).

Let us end the introduction with comments on related literature. To the best of our knowledge, the relation of absolute continuity/singularity and explosion of a timechanged process has not been reported before. We think that our new integral tests for 
absolute continuity/singularity and explosion/non-explosion illustrate that working out this connection is fruitful. The integral tests in $[9,29]$ for absolute continuity, singularity and the UI martingale property in one-dimensional frameworks follow from our result and Feller's test for explosion under additional assumptions on the coefficients. The existence of $\mathrm{E}(\mathrm{L}) \mathrm{MMs}$ for one- and multidimensional diffusion models with finite time horizon has, e.g., been studied in [10, 11, 30, 39]. Certain one-dimensional diffusion models with infinite time horizon have been studied in [30]. In this article we focus on multidimensional models. Beginning with [41], existence and uniqueness results for timechanged Markov processes have a long history, see, e.g., $[6,40]$ for more information. In most of the classical work, the function $\mathfrak{f}$ is assumed to be uniformly bounded away from zero, which implies that time-changes of conservative diffusions are themselves conservative. More general positive continuous $\mathfrak{f}$ are considered in the recent article [24] in combination with linear growth conditions for non-explosion. The novelty of our existence and uniqueness result is that we work without additional assumptions for non-explosion. This is crucial for the question of absolute continuity and singularity. Moreover, we work under sort of minimal assumptions on the original diffusion $P$ by assuming only existence and locally bounded coefficients.

The article is structured as follows: In Section 2 we present our main results, in Section 3 we discuss applications and in Section 4 we prove our main theorem. In Appendix B we recall the relation of martingale problems and weak solutions of stochastic differential equations, and in Appendix $\mathrm{C}$ we collect some existence and uniqueness results for solutions of martingale problems.

\section{Main results}

Let $\mathbb{R}_{\Delta}^{d} \triangleq \mathbb{R}^{d} \cup\{\Delta\}$ be the one-point compactification of $\mathbb{R}^{d}$ and let $\Omega$ be the space of all continuous functions $\mathbb{R}_{+} \rightarrow \mathbb{R}_{\Delta}^{d}$ which are absorbed in $\Delta$. Define $X$ to be the coordinate process on $\Omega$, i.e., $X_{t}(\omega)=\omega(t)$ for all $\omega \in \Omega$ and $t \in \mathbb{R}_{+}$, and define $\mathcal{F} \triangleq \sigma\left(X_{t}, t \in \mathbb{R}_{+}\right), \mathcal{F}_{t}^{o} \triangleq \sigma\left(X_{s}, s \in[0, t]\right)$ and $\mathcal{F}_{t} \triangleq \mathcal{F}_{t+}^{o} \triangleq \bigcap_{s>t} \mathcal{F}_{s}^{o}$ for all $t \in \mathbb{R}_{+}$. Except stated otherwise, all terms such as martingale, stopping time etc. correspond to $\mathbf{F} \triangleq\left(\mathcal{F}_{t}\right)_{t \geq 0}$ as underlying filtration. Let $\mathbb{S}^{d}$ be the space of symmetric non-negative definite real-valued $d \times d$ matrices.

For $n \in \mathbb{N}$ we set

$$
\theta_{n} \triangleq \inf \left(t \in \mathbb{R}_{+}:\left\|X_{t}\right\| \geq n\right), \quad \theta \triangleq \inf \left(t \in \mathbb{R}_{+}: X_{t}=\Delta\right)=\lim _{m \rightarrow \infty} \theta_{m} .
$$

It is well-known that $\theta_{n}$ and $\theta$ are stopping times. We fix two Borel functions $\mathfrak{b}: \mathbb{R}^{d} \rightarrow \mathbb{R}^{d}$ and $\mathfrak{a}: \mathbb{R}^{d} \rightarrow \mathbb{S}^{d}$ and assume the following:

(S1) $\mathfrak{b}$ and $\mathfrak{a}$ are locally bounded.

Here, $S$ is an acronym for standing, which indicates that the assumption is in force for the remainder of the section. For reader's convenience and because in some cases not all standing assumptions are needed, we indicate in every result which standing assumptions are used.

The following definition of a martingale problem is taken from [33], where it is called generalized martingale problem due to the possibility of explosion. For simplicity we drop the term generalized.

Definition 2.1. We say that a probability measure $P$ on $(\Omega, \mathcal{F})$ solves the martingale problem $M P\left(\mathfrak{a}, \mathfrak{b}, x_{0}\right)$, where $x_{0} \in \mathbb{R}^{d}$, if $P\left(X_{0}=x_{0}\right)=1$ and for all $n \in \mathbb{N}$ and $f \in C^{2}\left(\mathbb{R}^{d}\right)$ the process

$$
f\left(X_{\cdot \wedge \theta_{n}}\right)-f\left(x_{0}\right)-\int_{0}^{\cdot \wedge \theta_{n}}\left(\left\langle\nabla f\left(X_{s}\right), \mathfrak{b}\left(X_{s}\right)\right\rangle+\frac{1}{2} \operatorname{tr}\left(\nabla^{2} f\left(X_{s}\right) \mathfrak{a}\left(X_{s}\right)\right)\right) d s
$$


is a $P$-martingale. A solution $P$ is called conservative (or non-explosive), if $P(\theta=\infty)=1$, and almost surely explosive, if $P(\theta<\infty)=1$.

It is well-known that martingale problems have a one-to-one relation to weak solutions of stochastic differential equations, see, e.g., [17, Section 5.3] or [22, Section 5.5.B]. For reader's convenience, we recall this connection in Appendix B.

The following theorem is the key observation in this article. It shows that perpetual integrals are distributed as the explosion time of a time-changed diffusion.

Theorem 2.2. Assume (S1), let $\mathfrak{f}: \mathbb{R}^{d} \rightarrow(0, \infty)$ be Borel and locally bounded away from zero and infinity and let $x_{0} \in \mathbb{R}^{d}$. There exists a measurable map $V: \Omega \rightarrow \Omega$ such that for every solution $P_{x_{0}}$ to the $M P\left(\mathfrak{a}, \mathfrak{b}, x_{0}\right)$ the following hold:

(i) $P_{x_{0}}^{\circ} \triangleq P_{x_{0}} \circ V^{-1}$ solves the $M P\left(\mathfrak{f}^{-1} \mathfrak{a}, \mathfrak{f}^{-1} \mathfrak{b}, x_{0}\right)$.

(ii) For all Borel sets $A \subseteq[0, \infty]$

$$
P_{x_{0}}\left(\int_{0}^{\theta} \mathfrak{f}\left(X_{s}\right) d s \in A\right)=P_{x_{0}}^{\circ}(\theta \in A) .
$$

Moreover, if $P_{x_{0}}^{\circ}$ is the unique solution to the $M P\left(\mathfrak{f}^{-1} \mathfrak{a}, \mathfrak{f}^{-1} \mathfrak{b}, x_{0}\right)$, then $P_{x_{0}}$ is the unique solution to the $M P\left(\mathfrak{a}, \mathfrak{b}, x_{0}\right)$.

Remark 2.3. By symmetry, Theorem 2.2 yields that existence and uniqueness hold simultaneously for the MPs $\left(\mathfrak{a}, \mathfrak{b}, x_{0}\right)$ and $\left(\mathfrak{f}^{-1} \mathfrak{a}, \mathfrak{f}^{-1} \mathfrak{b}, x_{0}\right)$, i.e., one of these problems has a solution precisely in case the other has a solution and this solution is unique precisely if the other problem has a unique solution.

The proof of Theorem 2.2 is given in Section 4 below. Let us shortly explain the main idea: We first define a right-continuous measurable process $Y$ via a random time-change, i.e. we set

$$
Y_{t} \triangleq \begin{cases}X_{L_{t}}, & t<T_{\theta}, \\ \Delta, & t \geq T_{\theta},\end{cases}
$$

with

$$
T_{t} \triangleq \int_{0}^{t \wedge \theta} \mathfrak{f}\left(X_{s}\right) d s, \quad L_{t} \triangleq \inf \left(s \in \mathbb{R}_{+}: T_{s}>t\right), \quad t \in \mathbb{R}_{+} .
$$

The technical core in the proof of Theorem 2.2 is to show that $Y$ has almost surely continuous paths. This observation allows us to define the map $V$ as a modification of $Y$. Noting that $\theta(Y)=T_{\theta}$ explains (ii). To understand (i), consider the simplified case where $\mathfrak{f}$ is uniformly bounded away from zero and $P_{x_{0}}$ is conservative. Then, $P_{x_{0}}$-a.s.

$$
T_{\theta}=\int_{0}^{\infty} \mathfrak{f}\left(X_{s}\right) d s \geq \inf _{x \in \mathbb{R}^{d}} \mathfrak{f}(x) \int_{0}^{\infty} d s=\infty,
$$

and $P_{x_{0}}$-a.s. $Y=X_{L}$ has $\mathbb{R}^{d}$-valued continuous paths. Due to a change of variable, for every $f \in C^{2}\left(\mathbb{R}^{d}\right)$ we obtain that $P_{x_{0}}$-a.s.

$$
\begin{aligned}
\int_{0}^{\cdot}\left(\left\langle\nabla f\left(Y_{s}\right), \mathfrak{f}^{-1}\left(Y_{s}\right) \mathfrak{b}\left(Y_{s}\right)\right\rangle+\frac{1}{2} \operatorname{tr}\left(\nabla^{2} f\left(Y_{s}\right) \mathfrak{f}^{-1}\left(Y_{s}\right) \mathfrak{a}\left(Y_{s}\right)\right)\right) d s \\
=\int_{0}^{L .}\left(\left\langle\nabla f\left(X_{s}\right), \mathfrak{b}\left(X_{s}\right)\right\rangle+\frac{1}{2} \operatorname{tr}\left(\nabla^{2} f\left(X_{s}\right) \mathfrak{a}\left(X_{s}\right)\right)\right) d s .
\end{aligned}
$$

This observation allows us to deduce (i) from the optional stopping theorem. The assumption that $\mathfrak{f}$ is uniformly bounded away from zero simplifies the argument substantially. As we explain in Remark 2.7 below, a uniform boundedness assumption is typically too strong for our purpose. 
While we are mainly interested in (2.1), the existence and uniqueness parts of Theorem 2.2 are also useful, because they lead to localizations of known existence and uniqueness theorems. For example, if (S1) holds it follows from Theorem 2.2 and an existence result from [36] that existence of a solution to MP $\left(\mathfrak{a}, \mathfrak{b}, x_{0}\right)$ is implied by the weak ellipticity condition $\operatorname{det}(\mathfrak{a})^{-1} \in L_{\text {loc }}^{1}\left(\mathbb{R}^{d}\right)$. To see this, note first that there exists a continuous function $\mathfrak{f}: \mathbb{R}^{d} \rightarrow(0, \infty)$ such that $\mathfrak{f} \mathfrak{b}$ and $\mathfrak{f a}$ are bounded. Then, [36, Theorem 2] yields that the MP $\left(\mathfrak{f a}, \mathfrak{f} \mathfrak{b}, x_{0}\right)$ has a (conservative) solution. Finally, Theorem 2.2 implies that also the MP $\left(\mathfrak{a}, \mathfrak{b}, x_{0}\right)$ has a (not necessarily conservative) solution. We give more details on this in Appendix C.

Fix a third Borel function $\mathfrak{c}: \mathbb{R}^{d} \rightarrow \mathbb{R}^{d}$ and assume the following:

(S2) $\mathfrak{a c}$ is locally bounded.

Before we turn to our main application, we report a simple observation which we believe to be of interest: Absolute continuity and singularity are invariant under timechanges. We outline an application of this observation in Section 3.3.1 below.

Corollary 2.4. Assume that (S1) and (S2) hold and let $\mathfrak{f}: \mathbb{R}^{d} \rightarrow(0, \infty)$ be a Borel function which is locally bounded away from zero and infinity and take $x_{0} \in \mathbb{R}^{d}$. Further, let $P_{x_{0}}$ be the unique solution to the $M P\left(\mathfrak{a}, \mathfrak{b}, x_{0}\right)$, let $Q_{x_{0}}$ be the unique solution to the MP $\left(\mathfrak{a}, \mathfrak{b}+\mathfrak{a c}, x_{0}\right)$, let $P_{x_{0}}^{\circ}$ be the unique solution to the $M P\left(\mathfrak{f}^{-1} \mathfrak{a}, \mathfrak{f}^{-1} \mathfrak{b}, x_{0}\right)$ and let $Q_{x_{0}}^{\circ}$ be the unique solution to the $M P\left(\mathfrak{f}^{-1} \mathfrak{a}, \mathfrak{f}^{-1}(\mathfrak{b}+\mathfrak{a c}), x_{0}\right)$. The following hold:

(i) $P_{x_{0}} \ll Q_{x_{0}}$ if and only if $P_{x_{0}}^{\circ} \ll Q_{x_{0}}^{\circ}$.

(ii) $P_{x_{0}} \perp Q_{x_{0}}$ if and only if $P_{x_{0}}^{\circ} \perp Q_{x_{0}}^{\circ}$.

Proof. Let $V$ be as in Theorem 2.2, let $A \in \mathcal{F}$ be such that $Q_{x_{0}}^{\circ}(A)=0$ and set $B \triangleq$ $\{V \in A\} \in \mathcal{F}$. Then, Theorem 2.2 yields that $Q_{x_{0}}(B)=Q_{x_{0}}^{\circ}(A)=0$. Thus, $P_{x_{0}} \ll Q_{x_{0}}$ implies $P_{x_{0}}^{\circ}(A)=P_{x_{0}}(B)=0$ and consequently, $P_{x_{0}}^{\circ} \ll Q_{x_{0}}^{\circ}$. The converse implication in (i) follows by symmetry. Part (ii) can be shown in the same manner.

From now on, we also assume the following:

(S3) For every $x \in \mathbb{R}^{d}$ there exists a unique solution $P_{x}$ to the MP $(\mathfrak{a}, \mathfrak{b}, x)$, and for a fixed $x_{0} \in \mathbb{R}^{d}$ there exists a solution $Q_{x_{0}}$ to the MP $\left(\mathfrak{a}, \mathfrak{b}+\mathfrak{a c}, x_{0}\right)$.

Analytic conditions for (S3) are given in Proposition C.1 in Appendix C.

Next, we introduce a non-negative local $Q_{x_{0}}$-martingale which relates $Q_{x_{0}}$ and $P_{x_{0}}$. For this, we assume the following:

(S4) $\langle\mathfrak{c}, \mathfrak{a c}\rangle$ is locally bounded.

If (S1) - (S4) hold, $Q_{x_{0}}$ from (S3) is unique by Proposition C.3 in Appendix C. We set

$$
\bar{X}_{\cdot \wedge \theta_{n}} \triangleq X_{\cdot \wedge \theta_{n}}-X_{0}-\int_{0}^{\cdot \wedge \theta_{n}}\left(\mathfrak{b}\left(X_{s}\right)+\mathfrak{a}\left(X_{s}\right) \mathfrak{c}\left(X_{s}\right)\right) d s .
$$

By definition of the martingale problem, $\bar{X}_{\cdot \wedge \theta_{n}}$ is a continuous $Q_{x_{0}}$-martingale with quadratic variation process

$$
\left[\bar{X}_{\cdot \wedge \theta_{n}}, \bar{X}_{\cdot \wedge \theta_{n}}\right]=\int_{0}^{\cdot \wedge \theta_{n}} \mathfrak{a}\left(X_{s}\right) d s .
$$

By assumption (S4), the integral process $\bar{Y}_{\cdot \wedge \theta_{n}} \triangleq \int_{0}^{\cdot \wedge \theta_{n}}\left\langle\mathfrak{c}\left(X_{s}\right), d \bar{X}_{s}\right\rangle$ is well-defined as a continuous $Q_{x_{0}}$-martingale with quadratic variation process

$$
\left[\bar{Y}_{\cdot \wedge \theta_{n}}, \bar{Y}_{\cdot \wedge \theta_{n}}\right]=\int_{0}^{\cdot \wedge \theta_{n}}\left\langle\mathfrak{c}\left(X_{s}\right), \mathfrak{a}\left(X_{s}\right) \mathfrak{c}\left(X_{s}\right)\right\rangle d s .
$$


Lemma 2.5. Assume that (S1) - (S4) hold. Then, the process

$$
Z_{t} \triangleq \begin{cases}\exp \left(-\int_{0}^{t}\left\langle\mathfrak{c}\left(X_{s}\right), d \bar{X}_{s}\right\rangle-\frac{1}{2} \int_{0}^{t}\left\langle\mathfrak{c}\left(X_{s}\right), \mathfrak{a}\left(X_{s}\right) \mathfrak{c}\left(X_{s}\right)\right\rangle d s\right), & t<\theta \\ \liminf _{n \rightarrow \infty} Z_{\theta_{n}}, & t \geq \theta\end{cases}
$$

is a non-negative local $Q_{x_{0}}$-martingale and $Q_{x_{0}}$-a.s. the terminal value $Z_{\infty} \triangleq \lim _{t \rightarrow \infty} Z_{t}$ exists and is finite.

Proof. It follows similar to the proof of [20, Lemma 12.43] that $Z$ is a non-negative local $Q_{x_{0}}$-martingale. Thus, $Z$ is a non-negative $Q_{x_{0}}$-supermartingale by Fatou's lemma, and the existence of a finite terminal value follows from the supermartingale convergence theorem.

As in the introduction, we set

$$
A_{\theta} \triangleq \int_{0}^{\theta}\left\langle\mathfrak{c}\left(X_{s}\right), \mathfrak{a}\left(X_{s}\right) \mathfrak{c}\left(X_{s}\right)\right\rangle d s
$$

The next proposition is a version of [3, Theorem 1] for possibly non-conservative martingale problems. Closely related results are also given in [12, Corollaries 3.4 and 5.1] and [37, Theorem 3.3]. The setting in [12, 37] is not completely identical to ours, because the path space in $[12,37]$ allows also discontinuous explosion. This freedom is crucial for the extension arguments used in $[12,37]$. In Appendix A we explain how part (i) of the next proposition can be deduced from [12, Corollary 3.4]. The proof of (ii) is identical to those of [3, Theorem 1] and omitted.

Proposition 2.6. Assume that (S1) - (S4) hold.

(i) The following are equivalent:

(a) $P_{x_{0}} \ll Q_{x_{0}}$ with $\frac{d P_{x_{0}}}{d Q_{x_{0}}}=Z_{\infty}$.

(b) The local $Q_{x_{0}}$-martingale $Z$ as defined in (2.2) is a uniformly integrable (UI) $Q_{x_{0}}$-martingale.

(c) ${\stackrel{P}{x_{0}}}_{x_{0}}\left(A_{\theta}<\infty\right)=1$.

(ii) The following are equivalent:

(a) $P_{x_{0}} \perp Q_{x_{0}}$.

(b) $P_{x_{0}}\left(A_{\theta}=\infty\right)=1$.

From now on we also assume the following:

(S5) $\langle\mathfrak{c}, \mathfrak{a c}\rangle$ is locally bounded away from zero.

Remark 2.7. In case $\langle\mathfrak{c}, \mathfrak{a c}\rangle$ is uniformly bounded away from zero and $P_{x_{0}}$ is conservative, we have $P_{x_{0}}\left(A_{\theta}=\infty\right)=1$ and Proposition 2.6 shows that $P_{x_{0}} \perp Q_{x_{0}}$. This observation explains that a uniform boundedness assumption is too strong for a characterization of absolute continuity.

Due to Theorem 2.2, there exists a unique solution $P_{x_{0}}^{\circ}$ to the time-changed MP $\left(\langle\mathfrak{c}, \mathfrak{a c}\rangle^{-1} \mathfrak{a},\langle\mathfrak{c}, \mathfrak{a c}\rangle^{-1} \mathfrak{b}, x_{0}\right)$ and $P_{x_{0}}\left(A_{\theta}<\infty\right)=P_{x_{0}}^{\circ}(\theta<\infty)$. In view of Proposition 2.6, this observation allows us to relate absolute continuity and singularity of $Q_{x_{0}}$ and $P_{x_{0}}$ to almost sure (non-)explosion properties of $P_{x_{0}}^{\circ}$ :

Corollary 2.8. Assume that (S1) - (S5) hold.

(i) (i.a) - (i.c) in Proposition 2.6 are equivalent to $P_{x_{0}}^{\circ}(\theta<\infty)=1$.

(ii) (ii.a) and (ii.b) in Proposition 2.6 are equivalent to $P_{x_{0}}^{\circ}(\theta=\infty)=1$. 
Applications of Corollary 2.8 are discussed in Section 3 below. Next, we comment on the difference between finite and infinite time horizons. As we explain in Appendix A, the next proposition, which can be seen as a local version of Proposition 2.6 (i), follows from [12, Corollary 3.4], see also [12, Corollary 5.1] and [37, Theorem 3.3] for closely related statements.

Proposition 2.9. Suppose that (S1) - (S4) hold. The following are equivalent:

(i) $P_{x_{0}} \ll$ loc $Q_{x_{0}}$, i.e., $P_{x_{0}} \ll Q_{x_{0}}$ on $\mathcal{F}_{t}$ for all $t \in \mathbb{R}_{+}$.

(ii) The local $Q_{x_{0}}$-martingale $Z$ as defined in (2.2) is a $Q_{x_{0}}$-martingale.

(iii) $P_{x_{0}}$-a.s. $A_{\theta}<\infty$ on $\{\theta<\infty\}$.

As the next corollary shows, in case $Q_{x_{0}}$ is conservative local absolute continuity of $P_{x_{0}}$ and $Q_{x_{0}}$ and the martingale property of $Z$ are equivalent to non-explosion of $P_{x_{0}}$. This observation is well-known, see, e.g., [40, Exercise 10.3.2] or [7, 29, 39].

Corollary 2.10. Suppose that (S1) - (S4) hold and that $Q_{x_{0}}$ is conservative. The following are equivalent:

(i) $P_{x_{0}} \ll$ loc $Q_{x_{0}}$.

(ii) The local $Q_{x_{0}}$-martingale $Z$ is a $Q_{x_{0}}$-martingale.

(iii) $P_{x_{0}}$ is conservative.

Proof. The implications (iii) $\Rightarrow$ (i) $\Leftrightarrow$ (ii) follow directly from Proposition 2.9. If (i) holds, then $P_{x_{0}}(\theta \leq t)=0$ for all $t \in \mathbb{R}_{+}$, because $Q_{x_{0}}$ is conservative. This shows (i) $\Rightarrow$ (iii).

In view of Proposition 2.9 and Corollary 2.10, we observe an interesting difference between the characterizations of local absolute continuous and global absolute continuity, and between the martingale property and the UI martingale property of the local martingale $Z$ : The local absolute continuity $P_{x_{0}} \ll_{\text {loc }} Q_{x_{0}}$ and the $Q_{x_{0}}$-martingale property of $Z$ are related to non-explosion of $P_{x_{0}}$, while the absolute continuity $P_{x_{0}} \ll Q_{x_{0}}$ and the UI $Q_{x_{0}}$-martingale property of $Z$ are related to almost sure explosion of $P_{x_{0}}^{\circ}$. Moreover, Corollary 2.8 shows that absolute continuity and singularity can be related to the explosion of one auxiliary diffusion. This is not necessarily true for local absolute continuity and the martingale property, see Proposition 2.9 and [29, Remark (ii), p. 10].

Let us also comment on the role played by the initial value.

Lemma 2.11. Assume that $\mathfrak{b}$ is locally bounded, that $\mathfrak{a}$ is locally Hölder continuous and that $\langle\xi, \mathfrak{a}(x) \xi\rangle>0$ for all $x \in \mathbb{R}^{d}$ and $\xi \in \mathbb{R}^{d} \backslash\{0\}$. Then, for every $x_{0} \in \mathbb{R}^{d}$ there exists a unique solution $P_{x_{0}}$ to the $M P\left(\mathfrak{a}, \mathfrak{b}, x_{0}\right)$ and the following hold:

(i) $P_{x_{0}}(\theta=\infty)=1$ holds for all $x_{0} \in \mathbb{R}^{d}$ if it holds for some $x_{0} \in \mathbb{R}^{d}$.

(ii) $P_{x_{0}}(\theta<\infty)=1$ holds for all $x_{0} \in \mathbb{R}^{d}$ if it holds for some $x_{0} \in \mathbb{R}^{d}$.

Proof. The existence and uniqueness of $P_{x}$ follows from Proposition C.1 in Appendix C. The maximum principle ([4, Lemma 1.4]) implies that non-negative harmonic functions vanish at all points whenever they vanish at one point. Because $x \mapsto P_{x}(\theta<\infty)$ and $x \mapsto 1-P_{x}(\theta<\infty)$ are harmonic ([4, Lemma 1.2]), the claim follows.

For the conservative case the following observation is implied by [3, Corollary 1].

Corollary 2.12. Suppose that $\mathfrak{b}$ and $\mathfrak{a} \mathfrak{c}$ are locally bounded, that $\mathfrak{a}$ satisfies the assumptions from Lemma 2.11 and that $\langle\mathfrak{c}, \mathfrak{a c}\rangle$ is strictly positive and locally Hölder continuous. Then, for every $x_{0} \in \mathbb{R}^{d}$ there exist unique solutions $P_{x_{0}}$ and $Q_{x_{0}}$ to the MPs $\left(\mathfrak{a}, \mathfrak{b}, x_{0}\right)$ and $\left(\mathfrak{a}, \mathfrak{b}+\mathfrak{a c}, x_{0}\right)$, respectively. Moreover, the following hold:

(i) $P_{x_{0}} \ll Q_{x_{0}}$ holds for all $x_{0} \in \mathbb{R}^{d}$ if it holds for some $x_{0} \in \mathbb{R}^{d}$.

(ii) $P_{x_{0}} \perp Q_{x_{0}}$ holds for all $x_{0} \in \mathbb{R}^{d}$ if it holds for some $x_{0} \in \mathbb{R}^{d}$. 
In the following section we present three applications of our results. First, we derive a Khasminskii-type integral test for absolute continuity and singularity, second, we derive a Feller-type integral test for explosion of a multidimensional time-changed Brownian motion, and, third, we outline applications to mathematical finance.

\section{Three applications}

\subsection{A Khasminskii-test for absolute continuity/singularity}

For $d=1$ almost sure explosion and non-explosion can be characterized via analytic integral tests, see, e.g., [22, Theorem 5.5.29, Proposition 5.5.32]. In combination with Corollary 2.8 these characterizations lead to [9, Corollaries 5.1, 5.3] and [29, Theorem 2.3] under additional assumptions on the coefficients. Most importantly, the time-change argument requires $\mathfrak{c} \neq 0$, which is not needed in [9, 29]. In return, Corollary 2.8 can be applied independent of the dimension. Moreover, the characterization of absolute continuity and singularity via almost sure explosion and non-explosion is useful, because even for multidimensional diffusions many analytic conditions for almost sure (non-)explosion are known, see, e.g., [27, 33, 40]. In the following we use some of these conditions to formulate a Khasminskii-type integral test for $P_{x_{0}} \ll Q_{x_{0}}$ and $P_{x_{0}} \perp Q_{x_{0}}$.

Condition 3.1. There exist continuous functions $B:\left[\frac{1}{2}, \infty\right) \rightarrow \mathbb{R}$ and $A:\left[\frac{1}{2}, \infty\right) \rightarrow(0, \infty)$ such that for all $x \in \mathbb{R}^{d}:\|x\| \geq 1$

$$
\begin{aligned}
A\left(\frac{\|x\|^{2}}{2}\right) & \leq \frac{\langle x, \mathfrak{a}(x) x\rangle}{\langle\mathfrak{c}(x), \mathfrak{a}(x) \mathfrak{c}(x)\rangle}, \\
\langle x, \mathfrak{a}(x) x\rangle B\left(\frac{\|x\|^{2}}{2}\right) & \leq \operatorname{tr}(\mathfrak{a}(x))+2\langle x, \mathfrak{b}(x)\rangle,
\end{aligned}
$$

and

$$
\int_{\frac{1}{2}}^{\infty} \frac{1}{C(z)} \int_{\frac{1}{2}}^{z} \frac{C(u) d u}{A(u)} d z<\infty
$$

where

$$
C(z) \triangleq \exp \left(\int_{\frac{1}{2}}^{z} B(u) d u\right) .
$$

Condition 3.2. There exists an $R>0$ and continuous functions $B:[R, \infty) \rightarrow \mathbb{R}$ and $A:[R, \infty) \rightarrow(0, \infty)$ such that for all $x \in \mathbb{R}^{d}:\|x\| \geq \sqrt{2 R}$

$$
\begin{aligned}
A\left(\frac{\|x\|^{2}}{2}\right) & \geq \frac{\langle x, \mathfrak{a}(x) x\rangle}{\langle\mathfrak{c}(x), \mathfrak{a}(x) \mathfrak{c}(x)\rangle}, \\
\langle x, \mathfrak{a}(x) x\rangle B\left(\frac{\|x\|^{2}}{2}\right) & \geq \operatorname{tr}(\mathfrak{a}(x))+2\langle x, \mathfrak{b}(x)\rangle,
\end{aligned}
$$

and

where

$$
\int_{R}^{\infty} \frac{1}{C(z)} \int_{R}^{z} \frac{C(u) d u}{A(u)} d z=\infty
$$

$$
C(z) \triangleq \exp \left(\int_{R}^{z} B(u) d u\right)
$$

Corollary 3.3. Assume that (S1) - (S5) from Section 2 hold.

(i) Suppose that Condition 3.1 holds. Then, $P_{x_{0}} \ll Q_{x_{0}}$ with $\frac{d P_{x_{0}}}{d Q_{x_{0}}}=Z_{\infty}$. In particular, $Z$ as defined in (2.2) is a uniformly integrable $Q_{x_{0}}$-martingale.

(ii) Suppose that Condition 3.2 holds. Then, $P_{x_{0}} \perp Q_{x_{0}}$ and $Z$ as defined in (2.2) is no uniformly integrable $Q_{x_{0}}$-martingale. 
Proof. Due to [40, Theorem 10.2.4], Condition 3.1 implies that $P_{x_{0}}^{\circ}(\theta<\infty)=1$. In case Condition 3.2 holds, [40, Theorem 10.2.3] yields that $P_{x_{0}}^{\circ}(\theta=\infty)=1$. Now, all claims follow from Corollary 2.8 .

\subsection{An explosion-test for time-changed Brownian motion}

Let $\mathfrak{g}: \mathbb{R}^{d} \rightarrow(0, \infty)$ be a Borel function which is locally bounded away from zero and infinity. Due to Theorem 2.2, for every $x_{0} \in \mathbb{R}^{d}$ there exists a unique solution $P_{x_{0}}$ to the MP $\left(\mathfrak{g} \operatorname{Id}, 0, x_{0}\right)$. Providing an intuition, the probability measure $P_{x_{0}}$ is the (unique) law of the $\mathbb{R}_{\Delta}^{d}$-valued time-changed Brownian motion

$$
Y_{t} \triangleq \begin{cases}x_{0}+W_{L_{t}}, & t<T_{\infty}, \\ \Delta, & t \geq T_{\infty},\end{cases}
$$

where $W$ is a $d$-dimensional standard Brownian motion and

$$
T_{t} \triangleq \int_{0}^{t} \frac{d s}{\mathfrak{g}\left(x_{0}+W_{s}\right)}, \quad L_{t} \triangleq \inf \left(s \in \mathbb{R}_{+}: T_{s}>t\right), \quad t \in \mathbb{R}_{+} .
$$

Because Brownian motion is recurrent for $d=1,2$, Theorem 2.2 directly implies that $P_{x_{0}}$ is non-explosive in this case, see [31, Theorem 3.27] or [35, Proposition X.3.11]. We are interested in explosion properties of $P_{x_{0}}$ for the transient regime of Brownian motion.

For the remainder of this section let $d \geq 3$ and denote by $\mathcal{W}_{x_{0}}$ the $d$-dimensional Wiener measure with initial value $x_{0}$.

By the standard linear growth condition for non-explosion, we have $P_{x_{0}}(\theta=\infty)=1$ in case

$$
\mathfrak{g}(x) \leq \mathrm{C}(1+\|x\|)^{2}, \quad x \in \mathbb{R}^{d}, \mathrm{C}>0 .
$$

Using the Green kernel of Brownian motion, we also obtain a condition for almost sure explosion. More precisely, [31, Theorems 3.32, 3.33] yield that

$$
E^{\mathcal{W}_{x_{0}}}\left[\int_{0}^{\infty} \frac{d s}{\mathfrak{g}\left(X_{s}\right)}\right]=\mathrm{C}_{d} \int_{\mathbb{R}^{d}} \frac{\left\|x-x_{0}\right\|^{2-d} d x}{\mathfrak{g}(x)},
$$

for a dimension-dependent constant $C_{d}>0$. Together with Theorem 2.2 this observation implies the following:

Corollary 3.4. If $\int_{\mathbb{R}^{d}} \mathfrak{g}^{-1}(x)\left\|x-x_{0}\right\|^{2-d} d x<\infty$, then $E_{x_{0}}[\theta]<\infty$.

We now ask whether for certain choices of $\mathfrak{g}$ the convergence criterion in Corollary 3.4 is necessary for almost sure explosion. In other words, we ask whether in some cases $E_{x_{0}}[\theta]=\infty$ implies $P_{x_{0}}(\theta=\infty)>0$, which is of course in general not true. The following corollary shows that in case $\mathfrak{g}$ is locally Hölder continuous and at least of quadratic growth, $E_{x_{0}}[\theta]=\infty$ even implies $P_{x_{0}}(\theta=\infty)=1$.

Corollary 3.5. Suppose that $\mathfrak{g}$ is locally Hölder continuous and

$$
\mathfrak{g}(x) \geq \mathrm{C}(1+\|x\|)^{2}, \quad x \in \mathbb{R}^{d}, \mathrm{C}>0 .
$$

If $\int_{\mathbb{R}^{d}} \mathfrak{g}^{-1}(x)\|x\|^{2-d} d x=\infty$, then $P_{x_{0}}(\theta=\infty)=1$.

Proof. We define $\mathfrak{a} \triangleq \operatorname{Id}$ and $\mathfrak{c} \triangleq \mathfrak{g}^{-\frac{1}{2}} e_{1}$, where $e_{1}$ is the first unit vector. Let $Q_{x_{0}}$ be the unique solution to the MP $\left(\mathfrak{a}, \mathfrak{c}, x_{0}\right)$, see Proposition C.1 in Appendix C. Because $\mathfrak{a}$ and $\mathfrak{c}$ are bounded, the solution $Q_{x_{0}}$ is conservative. Note that $\langle\mathfrak{c}, \mathfrak{a c}\rangle=\mathfrak{g}^{-1}$ is a strictly positive continuous function. Corollary 2.8 yields that $P_{x_{0}}(\theta=\infty)=1$ if and only if $\mathcal{W}_{x_{0}} \perp Q_{x_{0}}$. It follows from [3, Corollary 4$]^{1}$ that

$$
\mathcal{W}_{x_{0}} \perp Q_{x_{0}} \Leftrightarrow \int_{\mathbb{R}^{d}} \frac{\|x\|^{2-d} d x}{\mathfrak{g}(x)}=\infty .
$$

\footnotetext{
${ }^{1}$ The statement of [3, Corollary 4] contains a small typo: $|b(x)|$ has to be replaced by $|b(x)|^{2}$, see [3, Eq. 1.2].
} 
This completes the proof.

The growth condition (3.1) and $\int_{\mathbb{R}^{d}} \mathfrak{g}^{-1}(x)\|x\|^{2-d} d x=\infty$ do not exclude each other: In case (3.1) holds, we have

$$
\int_{\mathbb{R}^{d}} \frac{\|x\|^{2-d} d x}{\mathfrak{g}(x)} \leq \mathrm{C} \int_{\mathbb{R}^{d}} \frac{\|x\|^{2-d} d x}{1+\|x\|^{2}}=\mathrm{C}_{d} \int_{0}^{\infty} \frac{r d r}{1+r^{2}}=\infty .
$$

The following proposition explains that in general the growth condition (3.1) is sharp. Proposition 3.6. Let $\rho: \mathbb{R}_{+} \rightarrow[1, \infty)$ be an increasing function with $\rho(0)=1$ and $\rho(x) \rightarrow \infty$ as $x \rightarrow \infty$. There exists a function $\mathfrak{g}$ such that the following hold:

(i) $\mathfrak{g}(x) \geq \frac{1+\|x\|^{2}}{\rho(\|x\|)}$ for all $x \in \mathbb{R}^{d}$.

(ii) $P_{0}(\theta<\infty)=1$.

(iii) $\int_{\mathbb{R}^{d}} \mathfrak{g}^{-1}(x)\|x\|^{2-d} d x=\infty$.

Proof. We adapt the proof of [3, Theorem 3]. Let $e_{1}$ be the first unit vector, set $x_{1} \triangleq e_{1}$ and define inductively

$$
R_{n} \triangleq 3^{-n}\left\|x_{n}\right\|, \quad x_{n+1} \in\left\{t e_{1}: t>4\left\|x_{n}\right\|, \rho\left(\frac{t}{2}\right)>4^{d(n+1)}\right\} .
$$

Set $B_{R}(x) \triangleq\left\{y \in \mathbb{R}^{d}:\|x-y\|<R\right\}$ and note that the balls $\left(B_{R_{n}}\left(x_{n}\right)\right)_{n \in \mathbb{N}}$ are disjoint, because

$$
\left\|x_{n+1}\right\|-\left\|x_{n}\right\|>\frac{3\left\|x_{n+1}\right\|}{4}=\frac{3^{n+2} R_{n+1}}{4}>\frac{9}{8}\left(R_{n+1}+R_{n}\right),
$$

where we use (3.2) and in particular that $3 R_{n+1}>4 R_{n}$. Define

$$
\mathfrak{g}(x) \triangleq \begin{cases}\frac{1+\|x\|^{2}}{\rho\left(\left\|x_{n}\right\|-R_{n}\right)}, & x \in B_{R_{n}}\left(x_{n}\right) \text { for some } n \in \mathbb{N} \\ 2+\|x\|^{4}, & x \notin \bigcup_{n \in \mathbb{N}} B_{R_{n}}\left(x_{n}\right) \triangleq G\end{cases}
$$

It is clear that $\mathfrak{g}$ is Borel and locally bounded away from zero and infinity. If $x \in B_{R_{n}}\left(x_{n}\right)$ we have $\left\|x_{n}\right\|-R_{n} \leq\|x\|$ and

$$
\frac{\mathfrak{g}(x) \rho(\|x\|)}{1+\|x\|^{2}}=\frac{\rho(\|x\|)}{\rho\left(\left\|x_{n}\right\|-R_{n}\right)} \geq 1,
$$

because $\rho$ is increasing. If $x \notin G=\bigcup_{n \in \mathbb{N}} B_{R_{n}}\left(x_{n}\right)$ we have

$$
\frac{\mathfrak{g}(x) \rho(\|x\|)}{1+\|x\|^{2}} \geq \rho(\|x\|) \geq 1 \text {. }
$$

In other words, (i) holds.

Next, we show (ii). Due to [31, Corollary 3.19] we have

$$
\sum_{n=1}^{\infty} \mathcal{W}_{0}\left(X \text { hits } B_{R_{n}}\left(x_{n}\right)\right)=\sum_{n=1}^{\infty}\left(\frac{R_{n}}{\left\|x_{n}\right\|}\right)^{d-2}=\sum_{n=1}^{\infty} 3^{-n(d-2)}<\infty
$$

Thus, the Borel-Cantelli lemma yields that $\mathcal{W}_{0}$-a.a. paths of $X$ hit only finitely many elements of $\left(B_{R_{n}}\left(x_{n}\right)\right)_{n \in \mathbb{N}}$. Recalling that Brownian motion is transient for $d \geq 3$, i.e., that $\mathcal{W}_{0}$-a.a. paths of $X$ leave bounded domains forever in finite time, we conclude that $\mathcal{W}_{0}$-a.s.

$$
\int_{0}^{\infty} \frac{\mathbb{I}_{G}\left(X_{s}\right) d s}{\mathfrak{g}\left(X_{s}\right)}<\infty
$$


Note that

$$
E^{\mathcal{W}_{0}}\left[\int_{0}^{\infty} \frac{d s}{2+\left\|X_{s}\right\|^{4}}\right]=\int_{\mathbb{R}^{d}} \frac{\|x\|^{2-d} d x}{2+\|x\|^{4}}=d \omega_{d} \int_{0}^{\infty} \frac{r d r}{2+r^{4}}<\infty,
$$

where $\omega_{d}$ is the volume of the unit ball in $\mathbb{R}^{d}$. We conclude that $\mathcal{W}_{0}$-a.s.

$$
\int_{0}^{\infty} \frac{d s}{\mathfrak{g}\left(X_{s}\right)}<\infty
$$

Thus, Theorem 2.2 yields that $P_{0}(\theta<\infty)=1$, i.e., (ii) holds.

It is left to verify (iii). Using (3.2), the fact that $f(x)=\|x\|^{2-d}$ is harmonic on $\mathbb{R}^{d} \backslash\{0\}$ and the mean-value property of harmonic functions, we obtain

$$
\begin{aligned}
\int_{\mathbb{R}^{d}} \frac{\|x\|^{2-d} d x}{\mathfrak{g}(x)} & \geq \sum_{n=1}^{\infty} \int_{B_{R_{n}}\left(x_{n}\right)} \frac{\|x\|^{2-d} d x}{\mathfrak{g}(x)} \\
& =\sum_{n=1}^{\infty} \rho\left(\left\|x_{n}\right\|-R_{n}\right) \int_{B_{R_{n}}\left(x_{n}\right)} \frac{\|x\|^{2-d} d x}{1+\|x\|^{2}} \\
& \geq \omega_{d} \sum_{n=1}^{\infty} \rho\left(\left\|x_{n}\right\|-R_{n}\right) \frac{\left\|x_{n}\right\|^{2-d} R_{n}^{d}}{1+\left(\left\|x_{n}\right\|+R_{n}\right)^{2}} \\
& \geq \omega_{d} \sum_{n=1}^{\infty} \frac{\rho\left(\left(1-3^{-n}\right)\left\|x_{n}\right\|\right)}{1+\left(1+3^{-n}\right)^{2}}\left(\frac{R_{n}}{\left\|x_{n}\right\|}\right)^{d} \\
& \geq \frac{\omega_{d}}{5} \sum_{n=1}^{\infty} \rho\left(\frac{\left\|x_{n}\right\|}{2}\right) 3^{-d n} \\
& \geq \frac{\omega_{d}}{5} \sum_{n=1}^{\infty}\left(\frac{4}{3}\right)^{d n}=\infty
\end{aligned}
$$

This implies (iii) and the proof is complete.

In case $\mathfrak{g}$ is a radial function, the growth condition on $\mathfrak{g}$ is not needed:

Corollary 3.7. Suppose that $\mathfrak{g}(x)=\mathfrak{s}(\|x\|)$ for a Borel function $\mathfrak{s}: \mathbb{R}_{+} \rightarrow(0, \infty)$ which is locally bounded away from zero and infinity. The following hold:

(i) If $\int_{\left\|x_{0}\right\|}^{\infty} r \mathfrak{s}^{-1}(r) d r<\infty$, then $P_{x_{0}}(\theta<\infty)=1$.

(ii) If $\int_{\left\|x_{0}\right\|}^{\infty} r \mathfrak{s}^{-1}(r) d r=\infty$, then $P_{x_{0}}(\theta=\infty)=1$.

Proof. Due to [16, Theorem 2] and [42, Corollary 3], for every locally bounded Borel function $\mathfrak{z}: \mathbb{R}_{+} \rightarrow \mathbb{R}_{+}$the following are equivalent:

(a) $\mathcal{W}_{x_{0}}\left(\int_{0}^{\infty} \mathfrak{z}\left(\left\|X_{s}\right\|\right) d s<\infty\right)>0$.

(b) $\mathcal{W}_{x_{0}}\left(\int_{0}^{\infty} \mathfrak{z}\left(\left\|X_{s}\right\|\right) d s<\infty\right)=1$.

(c) $\int_{\left\|x_{0}\right\|}^{\infty} z \mathfrak{z}(z) d z<\infty$.

The claims now follow directly from Theorem 2.2 .

Finally, we give a precise integrability condition, which is, in contrast to the conditions above, not completely analytic, because it involves probability via transient sets of Brownian motion. Let $\mathcal{T}$ be the collection of all sets $G \in \mathcal{B}\left(\mathbb{R}^{d}\right)$ such that $G^{c}$ is transient, i.e.,

$$
\mathcal{W}_{x}\left(X_{s} \in G \text { for all } s \in \mathbb{R}_{+}\right)>0 \text { for some } x \in \mathbb{R}^{d} \text {. }
$$

We stress that any Borel subset of $\mathbb{R}^{d}$ whose complement is bounded belongs to $\mathcal{T}$. However, there are examples of sets in $\mathcal{T}$ with unbounded complement, see the comment below the following corollary. For more comments on $\mathcal{T}$ we refer to [2, pp. $470-471$ ]. 
Corollary 3.8. Suppose that $\mathfrak{g}$ is locally Hölder continuous. The following are equivalent:

(i) $P_{x_{0}}(\theta=\infty)=1$.

(ii) $\int_{G} \mathfrak{g}^{-1}(x)\|x\|^{2-d} d x=\infty$ for all $G \in \mathcal{T}$.

Proof. According to [2, Proposition 3.1, Theorem 3.5], part (ii) is equivalent to

(iii) $\mathcal{W}_{x}\left(\int_{0}^{\infty} \mathfrak{g}^{-1}\left(X_{s}\right) d s=\infty\right)=1$ for Lebesgue a.a. $x \in \mathbb{R}^{d}$.

The claim now follows from Theorem 2.2 and Lemma 2.11.

Note that Proposition 3.6 is in line with Corollary 3.8: Let $G$ be as in the proof of Proposition 3.6. Then, (3.3) yields that

$$
\mathcal{W}_{0}\left(X_{s} \in G \text { for some } s \in \mathbb{R}_{+}\right) \leq \sum_{n=1}^{\infty} 3^{-n}=\frac{1}{2},
$$

which implies $G^{c} \in \mathcal{T}$. Thus, (ii) in Corollary 3.8 is violated in case of Proposition 3.6, because $\mathfrak{g}(x)=2+\|x\|^{4}$ on $G^{c}$ and therefore

$$
\int_{G^{c}} \frac{\|x\|^{2-d} d x}{\mathfrak{g}(x)} \leq \mathrm{C}_{d} \int_{0}^{\infty} \frac{r d r}{2+r^{4}}<\infty .
$$

\subsection{On the absence of arbitrage in diffusion markets}

In this section we outline applications to mathematical finance. We start by introducing a stochastic model for a financial market. Let $\mathfrak{a}: \mathbb{R}^{d} \rightarrow \mathbb{S}^{d}$ and $\mathfrak{b}: \mathbb{R}^{d} \rightarrow \mathbb{R}^{d}$ be locally bounded Borel functions and fix an initial value $x_{0} \in \mathbb{R}^{d}$. We assume that the MP $\left(\mathfrak{a}, \mathfrak{a b}, x_{0}\right)$ has a unique conservative solution $P_{x_{0}}$. In the following we consider $\left(\Omega, \mathcal{F}, \mathbf{F}, P_{x_{0}}\right)$ as underlying filtered probability space. Providing an intuition, possibly on an extension of $\left(\Omega, \mathcal{F}, \mathbf{F}, P_{x_{0}}\right)$, there exists a $d$-dimensional standard Brownian motion $B=\left(B^{1}, \ldots, B^{d}\right)$ such that

$$
d X_{t}=\mathfrak{a}^{\frac{1}{2}}\left(X_{t}\right)\left(d B_{t}+\mathfrak{a}^{\frac{1}{2}}\left(X_{t}\right) \mathfrak{b}\left(X_{t}\right) d t\right), \quad X_{0}=x_{0},
$$

see Proposition B.2 and Remark B.3 in Appendix B. For each $i=1, \ldots, d$ we define $S^{i}$ to be the stochastic exponential of $X^{i}$, i.e., the unique solution to the stochastic differential equation $d S_{t}^{i}=S_{t}^{i} d X_{t}^{i}$ with initial value $S_{0}=1$. We think of $S=\left(S^{1}, \ldots, S^{d}\right)$ as discounted price process in a financial market with $d$ risky assets.

It is an important question in mathematical finance whether there are certain arbitrage opportunities in the market. A probabilistic characterization of the existence is given by so-called fundamental theorems of asset pricing, which state that the absence of certain arbitrage opportunities is equivalent to the existence of a so-called equivalent (local) martingale measure $(E(L) M M)$, i.e., a probability measure which is on one hand equivalent (i.e., mutually absolutely continuous) to the real-world measure $P_{x_{0}}$ and on the other hand turns the discounted asset price process $S$ into a (local) UI martingale, see, e.g., [8, Corollary 5.2] and [15, Corollary 9.1.2]. Let us assume that the MP $\left(\mathfrak{a}, 0, x_{0}\right)$ has a unique solution $Q_{x_{0}}$. Because any equivalent change of measure preserves the quadratic variation, and continuous local martingales have necessarily zero drift, $Q_{x_{0}}$ is the only candidate for an ELMM. Clearly, because $P_{x_{0}}$ is assumed to be conservative, $Q_{x_{0}}$ is conservative whenever $P_{x_{0}}$ and $Q_{x_{0}}$ are equivalent. Consequently, we have the following:

Proposition 3.9. The following are equivalent:

(i) There exists an ELMM. 
(ii) $Q_{x_{0}}$ is an ELMM.

(iii) $Q_{x_{0}}$ is equivalent to $P_{x_{0}}$.

The plan for the next two subsections is the following: In Section 3.3.1 we consider diagonal diffusion coefficients and deduce analytic conditions for the existence of an ELMM from Corollary 2.4, Proposition 3.9 and results from [3]. In Section 3.3.2 we use Corollary 2.8 to formulate explosion criteria for an ELMM to be an EMM.

\subsubsection{On the existence of equivalent local martingale measures}

We assume that $\mathfrak{a}=\mathfrak{g}$ Id, where $\mathfrak{g}: \mathbb{R}^{d} \rightarrow(0, \infty)$ is locally bounded away from zero and infinity. In this case, (3.4) means that

$$
d X_{t}^{i}=\sqrt{\mathfrak{g}}\left(X_{t}\right)\left(d B_{t}^{i}+\sqrt{\mathfrak{g}}\left(X_{t}\right) \mathfrak{b}^{i}\left(X_{t}\right) d t\right), \quad X_{0}^{i}=x_{0}^{i}, \quad i=1, \ldots, d .
$$

Due to Proposition C.1 in Appendix C, there exists a unique solution $P_{x_{0}}^{\circ}$ to the MP $\left(\mathrm{Id}, \mathfrak{b}, x_{0}\right)$. Corollary 2.4 and Proposition 3.9 yield the following:

Corollary 3.10. (i) - (iii) from Proposition 3.9 hold if and only if $P_{x_{0}}^{\circ}$ is equivalent to the $d$-dimensional Wiener measure $\mathcal{W}_{x_{0}}$ with initial value $x_{0}$.

For a more general continuous setting with finite time horizon, a comparable characterization of the absence of arbitrage is given in [26]. More precisely, it is shown in [26, Proposition 2.3] that the existence of an ELMM is determined by the equivalence of a probability measure to the Wiener measure. Next, we use Corollary 3.10 to obtain analytic criteria for the existence of an ELMM. We start with a comment on the recurrent regime of Brownian motion. The Lebesgue measure is denoted by $\lambda$.

Corollary 3.11. If $d=1,2$, then an ELMM exists if and only if $\lambda$-a.e. $\mathfrak{b}=0$.

Proof. In case $\lambda(\mathfrak{b} \neq 0)=0$ we have $P_{x_{0}}^{\circ}=\mathcal{W}_{x_{0}}$ and Theorem 2.2 yields that the realworld measure $P_{x_{0}}$ is an ELMM. To see that $P_{x_{0}}^{\circ}=\mathcal{W}_{x_{0}}$, recall the uniqueness of $P_{x_{0}}^{\circ}$ and note that for any Borel function $f: \mathbb{R}^{d} \rightarrow \mathbb{R}_{+}$with $\lambda(f \neq 0)=0$

$$
E^{\mathcal{W}_{x_{0}}}\left[\int_{0}^{\infty} f\left(X_{s}\right) d s\right]=\int_{0}^{\infty} E^{\mathcal{W}_{x_{0}}}\left[f\left(X_{s}\right)\right] d s=0,
$$

because $X_{s}$ is normally distributed under $\mathcal{W}_{x_{0}}$.

If $\lambda(\mathfrak{b} \neq 0)>0$, then [35, Proposition X.3.11] yields $\mathcal{W}_{x_{0}}$-a.s. $\int_{0}^{\infty}\left\|\mathfrak{b}\left(X_{s}\right)\right\|^{2} d s=\infty$, and Proposition 2.6 and Corollary 3.10 show that no ELMM exists.

This observation is different for our exponential model and the (one-dimensional) diffusion model studied in [30] for which an ELMM might exist for non-trivial cases, see [30, Theorem 3.5].

We now consider the transient regime of Brownian motion. Corollary 3.10 and [3, Corollary 4] imply the following:

Corollary 3.12. Suppose that $d \geq 3$, that $\mathfrak{b}$ is locally Hölder continuous and that $\sup _{x \in \mathbb{R}^{d}}\|\mathfrak{b}(x)\|(1+\|x\|)<\infty$. Then, (i) - (iii) from Proposition 3.9 hold if and only if $\int_{\mathbb{R}^{d}}\|\mathfrak{b}(x)\|^{2}\|x\|^{2-d} d x<\infty$.

Of course, the assumption $\sup _{x \in \mathbb{R}^{d}}\|\mathfrak{b}(x)\|(1+\|x\|)<\infty$ implies that $\mathfrak{b}$ is bounded. While $P_{x_{0}}^{\circ}$ and $\mathcal{W}_{x_{0}}$ are locally equivalent whenever $\mathfrak{b}$ is bounded, this is not necessarily true for global equivalence. Indeed, the strong law of large numbers shows that the laws of Brownian motion with and without non-trivial linear drift are singular. This easy example also hints why we require $\|\mathfrak{b}(x)\| \rightarrow 0$ as $\|x\| \rightarrow \infty$. 


\subsubsection{On the existence of equivalent martingale measures}

Assume that $\mathfrak{b}=0$ and that $\mathfrak{a}$ is continuous and maps into the set of strictly positive definite $d \times d$ matrices. In this case, the real-world measure $P_{x_{0}}$ is already an ELMM and we ask for a condition when $P_{x_{0}}$ is even an EMM. Note that $S^{i}$ equals $Z$ as given in (2.2) when $-\mathfrak{c}$ is defined to be the $i$-th unit vector $e_{i}$. Thus, we are in the setting of Section 2 with $\mathfrak{b}$ set to be $\mathfrak{a} e_{i}$. In particular, (S1) - (S5) from Section 2 hold by Proposition C. 1 in Appendix $\mathrm{C}$ and the assumptions on $\mathfrak{a}$. Consequently, Corollary 2.8 implies the following:

Corollary 3.13. $S^{i}$ is a UI $Q_{x_{0}}$-martingale if and only if $Q_{x_{0}}^{i}(\theta<\infty)=1$, where $Q_{x_{0}}^{i}$ is the unique solution to the $\operatorname{MP}\left(\mathfrak{a}_{i i}^{-1} \mathfrak{a}, \mathfrak{a}_{i i}^{-1} \mathfrak{a} e_{i}, x_{0}\right)$.

Applying this corollary for all $i=1, \ldots, d$, we obtain an equivalent explosion test for $Q_{x_{0}}$ to be an EMM. Based on results from [27, 33, 40] one can also formulate analytic conditions.

We stress that the results for finite and infinite time horizons are quite different. For example, in case $d=1$ the probability measure $Q_{x_{0}}^{1}$ solves the MP $\left(1,1, x_{0}\right)$, which corresponds to Brownian motion with linear drift. Thus, $Q_{x_{0}}^{1}$ is conservative and consequently, $S=S^{1}$ is no UI $Q_{x_{0}}$-martingale, while it is a $Q_{x_{0}}$-martingale if and only if $\int_{0}^{\infty} \frac{d x}{\mathfrak{a}(x)}=\infty$, see [10, Proposition 5.2].

\section{Proof of Theorem 2.2}

In this section we prove Theorem 2.2, i.e., we prove the following:

Theorem. Assume (S1), let $\mathfrak{f}: \mathbb{R}^{d} \rightarrow(0, \infty)$ be Borel and locally bounded away from zero and infinity and let $x_{0} \in \mathbb{R}^{d}$. There exists a measurable map $V: \Omega \rightarrow \Omega$ such that for every solution $P_{x_{0}}$ to the $M P\left(\mathfrak{a}, \mathfrak{b}, x_{0}\right)$ the following hold:

(i) $P_{x_{0}}^{\circ} \triangleq P_{x_{0}} \circ V^{-1}$ solves the $M P\left(\mathfrak{f}^{-1} \mathfrak{a}, \mathfrak{f}^{-1} \mathfrak{b}, x_{0}\right)$.

(ii) For all Borel sets $A \subseteq[0, \infty]$

$$
P_{x_{0}}\left(\int_{0}^{\theta} \mathfrak{f}\left(X_{s}\right) d s \in A\right)=P_{x_{0}}^{\circ}(\theta \in A) .
$$

Moreover, if $P_{x_{0}}^{\circ}$ is the unique solution to the $M P\left(\mathfrak{f}^{-1} \mathfrak{a}, \mathfrak{f}^{-1} \mathfrak{b}, x_{0}\right)$, then $P_{x_{0}}$ is the unique solution to the $\operatorname{MP}\left(\mathfrak{a}, \mathfrak{b}, x_{0}\right)$.

Let $x_{0} \in \mathbb{R}^{d}$ and let $P_{x_{0}}$ be a solution to the MP $\left(\mathfrak{a}, \mathfrak{b}, x_{0}\right)$. To simplify the notation, we denote $P \equiv P_{x_{0}}$. We first define a right-continuous measurable process $Y$ via a random time-change. For $t \in \mathbb{R}_{+}$we set

$$
T_{t} \triangleq \int_{0}^{t \wedge \theta} \mathfrak{f}\left(X_{s}\right) d s, \quad L_{t} \triangleq \inf \left(s \in \mathbb{R}_{+}: T_{s}>t\right) .
$$

The functions $T, L: \mathbb{R}_{+} \rightarrow[0, \infty]$ are increasing. Because $\mathfrak{f}$ is locally bounded, we have $T_{\theta_{n} \wedge n}<\infty$ for all $n \in \mathbb{N}$. Using this and the strict positivity of $\mathfrak{f}$, we see that $T$ is finite, absolutely continuous and strictly increasing on $[0, \theta)$. Moreover, because $\lim _{t} \nearrow_{\theta} T_{t}=T_{\theta}$ by the monotone convergence theorem, $T$ is everywhere continuous. We also note that $L$ is finite, continuous and strictly increasing on $\left[0, T_{\theta}\right)$, everywhere right-continuous, and that $L_{T_{s}}=s$ for $s<\theta$ and $T_{L_{t}}=t$ for $t<T_{\theta}$, see [35, pp. $7-9$ ]. In particular, we have

$$
\lim _{t \nearrow T_{\theta}} L_{t}=\lim _{t \nearrow \theta} L_{T_{t}}=\theta
$$


For $t \in \mathbb{R}_{+}$we define

$$
Y_{t} \triangleq \begin{cases}X_{L_{t}}, & t<T_{\theta} \\ \Delta, & t \geq T_{\theta}\end{cases}
$$

It is easy to see that $Y$ is an $\mathbb{R}_{\Delta}^{d}$-valued right-continuous measurable process. Because $\left\{t<T_{\theta}\right\}=\left\{L_{t}<\theta\right\}$, we have $Y_{t} \in \mathbb{R}^{d}$ for every $t<T_{\theta}$ and consequently, $T_{\theta} \leq \theta(Y)$. Noting that $\theta(Y) \leq T_{\theta}$ by definition, we obtain that

$$
T_{\theta}=\theta(Y)=\inf \left(t \in \mathbb{R}_{+}: Y_{t}=\Delta\right)
$$

The next step is to show that $Y$ has almost surely continuous paths, i.e., that $P$-a.s. $Y_{T_{\theta}-}=\Delta$ on $\left\{T_{\theta}<\infty\right\}$. This observation allows us to modify $Y$ on a null set in order to define $V$ as in Theorem 2.2.

Discussion. On $\left\{T_{\theta}<\infty, \theta<\infty\right\}$ we simply have $Y_{T_{\theta}-}=X_{\theta}=\Delta$, but on $\left\{T_{\theta}<\infty, \theta=\right.$ $\infty\}$ it is necessary to understand the behavior of $X_{t}$ as $t \rightarrow \infty$. We stress that $\theta=\infty$ does not exclude $T_{\theta}<\infty$ in a pathwise sense. To see this, consider the following simple example:

$$
\mathfrak{f}(x)=\mathbb{I}_{(-\infty, 0)}(x)+\sum_{k=1}^{\infty} a_{k} \mathbb{I}_{[k-1, k)}(x), \quad x \in \mathbb{R}, 0<a_{k} \leq 1 .
$$

Clearly, $\mathfrak{f}$ is locally bounded away from zero and infinity and for $\omega(t)=t$ the integral

$$
\int_{0}^{\infty} \mathfrak{f}\left(X_{s}(\omega)\right) d s=\sum_{k=1}^{\infty} a_{k}
$$

converges or diverges depending on whether $\left(a_{k}\right)_{k \in \mathbb{N}}$ is summable or not. To understand why $P$-a.s. $Y_{T_{\theta}-}=\Delta$ on $\left\{T_{\theta}<\infty\right\}$ holds, note that problems with the limit of $X_{t}$ as $t \rightarrow \infty$ occur for paths which either stay in a bounded subset of $\mathbb{R}^{d}$ or have a recurrent behavior, where we think for instance of a one-dimensional Brownian path. These cases are excluded by considering the set $\left\{T_{\theta}<\infty\right\}$, because for some compact set $U \subset \mathbb{R}^{d}$ the positive value $\inf _{x \in U} \mathfrak{f}(x)$ will contribute to $T_{\theta}$ for an infinite time.

For every $n, m \in \mathbb{N}$ we define

$$
\begin{array}{clrl}
\sigma_{1}^{m} \triangleq 0, & \tau_{1}^{m} \triangleq \inf \left(t \in \mathbb{R}_{+}:\left\|X_{t}\right\| \geq m+1\right), \\
\sigma_{n+1}^{m} \triangleq \inf \left(t>\tau_{n}^{m}:\left\|X_{t}\right\| \leq m\right), & \tau_{n+1}^{m} \triangleq \inf \left(t>\sigma_{n+1}^{m}:\left\|X_{t}\right\| \geq m+1\right) .
\end{array}
$$

Set

$$
\mathcal{O} \triangleq \bigcap_{m=1}^{\infty} \bigcup_{n=1}^{\infty}\left\{\tau_{n}^{m}<\infty, \sigma_{n+1}^{m}=\infty\right\}
$$

and note that $\mathcal{O} \subseteq\left\{Y_{T_{\theta}-}=\Delta\right\}=\left\{X_{\theta-}=\Delta\right\}$. The proof of the following lemma borrows ideas from [19, Lemma IV.2.1].

Lemma 4.1. $P$-a.s. $\mathcal{O}^{c} \subseteq\left\{T_{\theta}=\infty\right\}$. 
Absolute continuity/singularity of multidimensional diffusions

Proof. We obtain

$$
\begin{aligned}
\mathcal{O}^{c} & =\bigcup_{m=1}^{\infty} \bigcap_{n=1}^{\infty}\left(\left\{\tau_{n}^{m}=\infty\right\} \cup\left\{\sigma_{n+1}^{m}<\infty\right\}\right) \\
& =\bigcup_{m=1}^{\infty} \bigcap_{n=1}^{\infty}\left(\left\{\tau_{n}^{m}=\infty, \sigma_{n}^{m}<\infty\right\} \cup\left\{\sigma_{n}^{m}=\infty\right\} \cup\left\{\sigma_{n+1}^{m}<\infty\right\}\right) \\
& \subseteq \bigcup_{m=1}^{\infty}\left(\left(\bigcup_{k=1}^{\infty}\left\{\tau_{k}^{m}=\infty, \sigma_{k}^{m}<\infty\right\}\right) \cup\left(\bigcap_{n=1}^{\infty}\left(\left\{\sigma_{n}^{m}=\infty\right\} \cup\left\{\sigma_{n+1}^{m}<\infty\right\}\right)\right)\right) \\
& =\bigcup_{m=1}^{\infty}\left(\left(\bigcup_{k=1}^{\infty}\left\{\tau_{k}^{m}=\infty, \sigma_{k}^{m}<\infty\right\}\right) \cup\left(\bigcap_{n=1}^{\infty}\left\{\sigma_{n}^{m}<\infty\right\}\right)\right) \\
& \subseteq\left(\bigcup_{m=1}^{\infty} \bigcup_{k=1}^{\infty}\left\{\tau_{k}^{m}=\infty, \sigma_{k}^{m}<\infty\right\}\right) \cup\left(\bigcup_{i=1}^{\infty} \bigcap_{n=1}^{\infty}\left\{\sigma_{n}^{i}<\infty\right\}\right) \\
& \triangleq \mathcal{O}_{1} \cup \mathcal{O}_{2} .
\end{aligned}
$$

Take $\omega \in \mathcal{O}_{1}$. Then, there exist $m=m(\omega), n=n(\omega) \in \mathbb{N}$ such that $\sigma_{n}^{m}(\omega)<\infty$ and $\left\|X_{t}(\omega)\right\| \leq m+1$ for all $t \geq \sigma_{n}^{m}(\omega)$. Consequently, $\theta(\omega)=\infty$ and

$$
T_{\theta(\omega)}(\omega)=\int_{0}^{\infty} \mathfrak{f}\left(X_{s}(\omega)\right) d s \geq \int_{\sigma_{n}(\omega)}^{\infty} \mathfrak{f}\left(X_{s}(\omega)\right) d s \geq \inf _{\|y\| \leq m+1} \mathfrak{f}(y) \int_{\sigma_{n}(\omega)}^{\infty} d s=\infty .
$$

This implies $\mathcal{O}_{1} \subseteq\left\{T_{\theta}=\infty\right\}$.

Set

$$
\Theta \triangleq \bigcup_{m=1}^{\infty}\left\{\sigma_{n}^{m}<\infty \text { for all } n \in \mathbb{N} \text { and } \sum_{k=1}^{\infty}\left(\tau_{k}^{m}-\sigma_{k}^{m}\right)=\infty\right\}
$$

Take $\omega \in \Theta$ and let $m=m(\omega) \in \mathbb{N}$ be as in the definition of $\Theta$. Then,

$$
T_{\theta(\omega)}(\omega) \geq \sum_{k=1}^{\infty} \int_{\sigma_{k}^{m}(\omega)}^{\tau_{k}^{m}(\omega)} \mathfrak{f}\left(X_{s}(\omega)\right) d s \geq \inf _{\|y\| \leq m+1} \mathfrak{f}(y) \sum_{k=1}^{\infty}\left(\tau_{k}^{m}(\omega)-\sigma_{k}^{m}(\omega)\right)=\infty
$$

This implies that $\Theta \subseteq\left\{T_{\theta}=\infty\right\}$.

Next, we show that $P$-a.s. $\mathcal{O}_{2}=\Theta$, which then implies that $P$-a.s. $\mathcal{O}^{c} \subseteq\left\{T_{\theta}=\infty\right\}$ and thereby completes the proof. We fix $m, n \in \mathbb{N}$. Clearly, we have on $\left\{\sigma_{n}^{m}<\infty\right\}$

$$
\tau_{n}^{m}-\sigma_{n}^{m}=\inf \left(t \in \mathbb{R}_{+}:\left\|X_{t+\sigma_{n}^{m}}\right\| \geq m+1\right) \triangleq \gamma .
$$

We set

$$
K_{t} \triangleq\left\|X_{t}\right\|^{2}-\left\|X_{0}\right\|^{2}-\int_{0}^{t}\left(2\left\langle X_{s}, \mathfrak{b}\left(X_{s}\right)\right\rangle+\operatorname{tr}\left(\mathfrak{a}\left(X_{s}\right)\right)\right) d s, \quad t<\theta,
$$

and on $\left\{\sigma_{n}^{m}<\infty\right\}$ we define

$$
M \triangleq K_{\cdot \wedge \gamma+\sigma_{n}^{m}}-K_{\sigma_{n}^{m}}, \quad I \triangleq \int_{\sigma_{n}^{m}}^{\cdot \wedge \gamma+\sigma_{n}^{m}}\left(2\left\langle X_{s}, \mathfrak{b}\left(X_{s}\right)\right\rangle+\operatorname{tr}\left(\mathfrak{a}\left(X_{s}\right)\right)\right) d s .
$$

Using that for every $t \in \mathbb{R}_{+}$on $\left\{\sigma_{n}^{m}<\infty\right\}$

$$
\left\{\left\|X_{t \wedge \gamma+\sigma_{n}^{m}}\right\| \geq m+1\right\} \subseteq\left\{\left|M_{t}\right| \geq \frac{1}{2}\right\} \cup\left\{\left|I_{t}\right| \geq \frac{1}{2}\right\},
$$

we obtain that

$$
\gamma \geq \inf \left(t \in \mathbb{R}_{+}:\left|M_{t}\right| \geq \frac{1}{2}\right) \wedge \inf \left(t \in \mathbb{R}_{+}:\left|I_{t}\right| \geq \frac{1}{2}\right) \text { on }\left\{\sigma_{n}^{m}<\infty\right\}
$$


Because

$$
\left|I_{t}\right| \leq\left(\sup _{\|y\| \leq m+1}|2\langle y, \mathfrak{b}(y)\rangle+\operatorname{tr}(\mathfrak{a}(y))| \vee 1\right) t \triangleq \alpha t \text { on }\left\{\sigma_{n}^{m}<\infty\right\},
$$

we obtain that

$$
\inf \left(t \in \mathbb{R}_{+}:\left|I_{t}\right| \geq \frac{1}{2}\right) \geq \frac{1}{2 \alpha} \text { on }\left\{\sigma_{n}^{m}<\infty\right\} .
$$

For every $t \in \mathbb{R}_{+}$we have $t \wedge \gamma+\sigma_{n}^{m}<\theta$ on $\left\{\sigma_{n}^{m}<\infty\right\}$. Consequently,

$$
\left(t \wedge \gamma+\sigma_{n}^{m}\right) \wedge \theta_{k} \wedge k \nearrow t \wedge \gamma+\sigma_{n}^{m} \text { as } k \rightarrow \infty \text { on }\left\{\sigma_{n}^{m}<\infty\right\} .
$$

Applying the definition of the martingale problem with $f(x)=\|x\|^{2}$ yields that for every $k \in \mathbb{N}$ the process $K_{. \wedge \theta_{k} \wedge k}$ is a $P$-martingale. Note that for every $t \in \mathbb{R}_{+}$

$$
\sup _{k \in \mathbb{N}}\left|K_{\left(t \wedge \gamma+\sigma_{n}^{m}\right) \wedge \theta_{k} \wedge k}-K_{\sigma_{n}^{m} \wedge \theta_{k} \wedge k}\right| \mathbb{I}_{\left\{\sigma_{n}^{m}<\infty\right\}} \leq 2(m+1)^{2}+\alpha t .
$$

It is well-known that $\sigma_{n}^{m}$ and $\tau_{n}^{m}$ are $\left(\mathcal{F}_{t}^{o}\right)_{t \geq 0}$-stopping times, see [17, Proposition 2.1.5]. We note that $t \wedge \gamma+\sigma_{n}^{m}$, which is set to be $\infty$ in case $\sigma_{n}^{m}=\infty$, is an $\left(\mathcal{F}_{t}^{o}\right)_{t \geq 0}$-stopping time, too. To see this, note that for all $s \in \mathbb{R}_{+}$

$$
\begin{aligned}
\left\{t \wedge \gamma+\sigma_{n}^{m} \leq s\right\}=\left\{t+\sigma_{n}^{m} \leq s,\right. & \left.\sigma_{n}^{m}<\infty, t+\sigma_{n}^{m} \leq \tau_{n}^{m}\right\} \\
& \cup\left\{\tau_{n}^{m} \leq s, \sigma_{n}^{m}<\infty, \tau_{n}^{m} \leq t+\sigma_{n}^{m}\right\} \in \mathcal{F}_{s}^{o},
\end{aligned}
$$

which holds due to the following facts: For any $\left(\mathcal{F}_{t}^{o}\right)_{t \geq 0}$-stopping times $\rho$ and $\tau$ it holds that $\mathcal{F}_{\rho}^{o} \cap\{\rho \leq s\} \subseteq \mathcal{F}_{s}^{o},\{\rho \leq \tau\} \in \mathcal{F}_{\rho}^{o} \cap \mathcal{F}_{\tau}^{o}$, and $\mathcal{F}_{\rho}^{o} \subseteq \mathcal{F}_{\tau}^{o}$ whenever $\rho \leq \tau$.

Let $s<t$ and take $A \in \overline{\mathcal{F}}_{s+\sigma_{n}^{m}}^{o}$ and $G \in \mathcal{F}_{\sigma_{n}^{m}}^{o}$. Recalling (4.4) and (4.5), the dominated convergence and the optional stopping theorem yield that

$$
\begin{aligned}
E^{P}\left[M_{t} \mathbb{I}_{A} \mathbb{I}_{G} \mathbb{I}_{\left\{\sigma_{n}^{m}<\infty\right\}}\right] \\
\quad=\lim _{k \rightarrow \infty} E^{P}\left[\left(K_{\left(t \wedge \gamma+\sigma_{n}^{m}\right) \wedge \theta_{k} \wedge k}-K_{\sigma_{n}^{m} \wedge \theta_{k} \wedge k}\right) \mathbb{I}_{A} \mathbb{I}_{G} \mathbb{I}_{\left\{\sigma_{n}^{m}<\infty\right\}}\right] \\
\quad=\lim _{k \rightarrow \infty} E^{P}\left[\left(E^{P}\left[K_{\left(t \wedge \gamma+\sigma_{n}^{m}\right) \wedge \theta_{k} \wedge k} \mid \mathcal{F}_{s+\sigma_{n}^{m}}\right]-K_{\sigma_{n}^{m} \wedge \theta_{k} \wedge k}\right) \mathbb{I}_{A} \mathbb{I}_{G} \mathbb{I}_{\left\{\sigma_{n}^{m}<\infty\right\}}\right] \\
=\lim _{k \rightarrow \infty} E^{P}\left[\left(K_{\left(s \wedge \gamma+\sigma_{n}^{m}\right) \wedge \theta_{k} \wedge k}-K_{\sigma_{n}^{m} \wedge \theta_{k} \wedge k}\right) \mathbb{I}_{A} \mathbb{I}_{G} \mathbb{I}_{\left\{\sigma_{n}^{m}<\infty\right\}}\right] \\
=E^{P}\left[M_{s} \mathbb{I}_{A} \mathbb{I}_{G} \mathbb{I}_{\left\{\sigma_{n}^{m}<\infty\right\}}\right] .
\end{aligned}
$$

We conclude the existence of a $P$-null set $N(s, t, A)$ such that

$$
E^{P}\left[\left(M_{t}-M_{s}\right) \mathbb{I}_{A} \mathbb{I}_{\left\{\sigma_{n}^{m}<\infty\right\}} \mid \mathcal{F}_{\sigma_{n}^{m}}^{o}\right](\omega)=0
$$

for all $\omega \notin N(s, t, A)$. Because $\mathcal{F}_{s+\sigma_{n}^{m}}^{o}=\sigma\left(X_{t \wedge\left(s+\sigma_{n}^{m}\right)}, t \in \mathbb{Q}_{+}\right)$, see [38, Theorem I.6], there exists a countable system $\mathcal{C}_{s}$ of generators of $\mathcal{F}_{s+\sigma_{n}^{m}}^{o}$. Set

$$
N \triangleq \bigcup_{t \in \mathbb{Q}_{+}} \bigcup_{\mathbb{Q}_{+}} \bigcup_{s<t} N(s, t, A),
$$

which is a $P$-null set. Now, we conclude that for all $\omega \notin N \cup\left\{\sigma_{n}^{m}=\infty\right\}$ the process $M$ is a continuous $P\left(\cdot \mid \mathcal{F}_{\sigma_{n}^{m}}^{o}\right)(\omega)$-martingale for the shifted filtration $\left(\mathcal{F}_{t+\sigma_{n}^{m}}^{o}\right)_{t \geq 0}$ and, by the backwards martingale convergence theorem, also for its right-continuous version $\mathbf{F}_{\sigma_{n}^{m}} \triangleq\left(\mathcal{F}_{t+\sigma_{n}^{m}}\right)_{t \geq 0}$, see also [21, Lemma 6.2].

Fix $\omega \notin N \cup\left\{\sigma_{n}^{m}=\infty\right\}$. It follows similar to the proof of [35, Proposition VIII.3.3] that $P\left(\cdot \mid \mathcal{F}_{\sigma_{n}^{m}}^{o}\right)(\omega)$-a.s.

$$
[M, M]=4 \int_{\sigma_{n}^{m}}^{\cdot \wedge \gamma+\sigma_{n}^{m}}\left\langle X_{s}, \mathfrak{a}\left(X_{s}\right) X_{s}\right\rangle d s
$$


The Dambis, Dubins-Schwarz theorem (see, e.g., [21, Theorem 16.4]) yields that on a standard extension of the filtered probability space $\left(\Omega, \mathcal{F}, \mathbf{F}_{\sigma_{n}^{m}}, P\left(\cdot \mid \mathcal{F}_{\sigma_{n}^{m}}^{o}\right)(\omega)\right)$, which we ignore in our notation for simplicity, there exists a one-dimensional Brownian motion $B$ such that $P\left(\cdot \mid \mathcal{F}_{\sigma_{n}^{m}}^{o}\right)(\omega)$-a.s. $M=B_{[M, M]}$. Because $P\left(\cdot \mid \mathcal{F}_{\sigma_{n}^{m}}^{o}\right)(\omega)$-a.s.

$$
4 \int_{\sigma_{n}^{m}}^{t \wedge \gamma+\sigma_{n}^{m}}\left\langle X_{s}, \mathfrak{a}\left(X_{s}\right) X_{s}\right\rangle d s \leq 4\left(\sup _{\|y\| \leq m+1}\langle y, \mathfrak{a}(y) y\rangle \vee 1\right) t \triangleq \beta t, \quad t \in \mathbb{R}_{+},
$$

we have $P\left(\cdot \mid \mathcal{F}_{\sigma_{n}^{m}}^{o}\right)(\omega)$-a.s.

$$
\inf \left(t \in \mathbb{R}_{+}:\left|B_{[M, M]_{t}}\right| \geq \frac{1}{2}\right) \geq \frac{\inf \left(t \in \mathbb{R}_{+}:\left|B_{t}\right| \geq \frac{1}{2}\right)}{\beta} \triangleq \frac{\tau}{\beta} .
$$

In summary, (4.2), (4.3) and (4.6) imply that

$$
E^{P}\left[e^{-\left(\tau_{n}^{m}-\sigma_{n}^{m}\right)} \mid \mathcal{F}_{\sigma_{n}^{m}}^{o}\right](\omega) \leq E\left[e^{-\frac{\tau}{\beta} \wedge \frac{1}{2 \alpha}}\right] \triangleq \mathrm{C} .
$$

We note that the law of $\tau$ under $P\left(\cdot \mid \mathcal{F}_{\sigma_{n}^{m}}^{o}\right)(\omega)$ only depends on the Wiener measure, which means that $C$ is a constant independent of $n, m$ and $\omega$. Note also that $C<1$. Now, we obtain for all $n \in \mathbb{Z}_{+}$

$$
\begin{aligned}
& E^{P}\left[\prod_{k=1}^{n+1} \mathbb{I}_{\left\{\sigma_{k}^{m}<\infty\right\}} e^{-\left(\tau_{k}^{m}-\sigma_{k}^{m}\right)}\right] \\
& \quad=E^{P}\left[\mathbb{I}_{\left\{\sigma_{n+1}^{m}<\infty\right\}} E^{P}\left[e^{-\left(\tau_{n+1}^{m}-\sigma_{n+1}^{m}\right)} \mid \mathcal{F}_{\sigma_{n+1}^{m}}^{o}\right] \prod_{k=1}^{n} \mathbb{I}_{\left\{\sigma_{k}^{m}<\infty\right\}} e^{-\left(\tau_{k}^{m}-\sigma_{k}^{m}\right)}\right] \\
& \quad \leq \mathrm{C} E^{P}\left[\prod_{k=1}^{n} \mathbb{I}_{\left\{\sigma_{k}^{m}<\infty\right\}} e^{-\left(\tau_{k}^{m}-\sigma_{k}^{m}\right)}\right]
\end{aligned}
$$

By induction, we conclude

$$
E^{P}\left[\prod_{k=1}^{n} \mathbb{I}_{\left\{\sigma_{k}^{m}<\infty\right\}} e^{-\left(\tau_{k}^{m}-\sigma_{k}^{m}\right)}\right] \leq \mathrm{C}^{n}, \quad n \in \mathbb{N} .
$$

Letting $n \rightarrow \infty$ and using the dominated convergence theorem yields that

$$
E^{P}\left[\prod_{k=1}^{\infty} \mathbb{I}_{\left\{\sigma_{k}^{m}<\infty\right\}} e^{-\left(\tau_{k}^{m}-\sigma_{k}^{m}\right)}\right]=0
$$

This implies that $P$-a.s. for all $m \in \mathbb{N}$

$$
e^{-\sum_{i=1}^{\infty}\left(\tau_{i}^{m}-\sigma_{i}^{m}\right)} \prod_{k=1}^{\infty} \mathbb{I}_{\left\{\sigma_{k}^{m}<\infty\right\}}=\prod_{k=1}^{\infty} \mathbb{I}_{\left\{\sigma_{k}^{m}<\infty\right\}} e^{-\left(\tau_{k}^{m}-\sigma_{k}^{m}\right)}=0 .
$$

We conclude that $P$-a.s. $\mathcal{O}_{2}=\Theta$. The proof is complete.

Remark 4.2. In case the $\operatorname{MP}(\mathfrak{a}, \mathfrak{b}, x)$ has a unique solution $P_{x}$ for all $x \in \mathbb{R}^{d}$ and $x \mapsto P_{x}$ is continuous, the proof of $P$-a.s. $\mathcal{O}_{2}=\Theta$ in Lemma 4.1 simplifies substantially: We equip $\Omega$ with the usual local uniform topology, which renders it into a Polish space, see [33, pp. $33-34$ ] for details. Because $\omega \mapsto \tau_{1}^{m}(\omega)$ is lower semi-continuous (see [33, Exercise 2.2.1]), the map $\omega \mapsto e^{-\tau_{1}^{m}(\omega)}$ is upper semi-continuous. Thus, [1, Theorem 15.5] yields that also $x \mapsto E_{x}\left[e^{-\tau_{1}^{m}}\right]$ is upper semi-continuous. Consequently, because on compact sets upper semi-continuous functions attain a maximum value, $\mathrm{C} \triangleq \sup _{\|x\| \leq m} E_{x}\left[e^{-\tau_{1}^{m}}\right]<1$. 
Now, using the strong Markov property, which is implied by uniqueness of $\left(P_{x}\right)_{x \in \mathbb{R}^{d}}$, we obtain

$$
\begin{aligned}
E_{x_{0}}\left[\prod_{k=1}^{n+1}\right. & \left.\mathbb{I}_{\left\{\sigma_{k}^{m}<\infty\right\}} e^{-\left(\tau_{k}^{m}-\sigma_{k}^{m}\right)}\right] \\
& =E_{x_{0}}\left[\mathbb{I}_{\left\{\sigma_{n+1}^{m}<\infty\right\}} E_{x_{0}}\left[e^{-\left(\tau_{n+1}^{m}-\sigma_{n+1}^{m}\right)} \mid \mathcal{F}_{\sigma_{n+1}^{m}}^{o}\right] \prod_{k=1}^{n} \mathbb{I}_{\left\{\sigma_{k}^{m}<\infty\right\}} e^{-\left(\tau_{k}^{m}-\sigma_{k}^{m}\right)}\right] \\
& =E_{x_{0}}\left[\mathbb{I}_{\left\{\sigma_{n+1}^{m}<\infty\right\}} E_{X_{\sigma_{n+1}^{m}}}\left[e^{-\tau_{1}^{m}}\right] \prod_{k=1}^{n} \mathbb{I}_{\left\{\sigma_{k}^{m}<\infty\right\}} e^{-\left(\tau_{k}^{m}-\sigma_{k}^{m}\right)}\right] \\
& \leq \mathrm{C} E_{x_{0}}\left[\prod_{k=1}^{n} \mathbb{I}_{\left\{\sigma_{k}^{m}<\infty\right\}} e^{-\left(\tau_{k}^{m}-\sigma_{k}^{m}\right)}\right] \leq \mathrm{C}^{n+1} \rightarrow 0 \text { as } n \rightarrow \infty
\end{aligned}
$$

The previous proof of Lemma 4.1 requires no uniqueness assumption on $P$ and no continuity assumptions on $\mathfrak{b}$ and/or $\mathfrak{a}$. Latter are often imposed to obtain continuity of $x \mapsto P_{x}$, see, for instance, [33, 40].

We now set

$$
V \triangleq \begin{cases}Y, & \text { on }\left\{T_{\theta}=\infty\right\} \cup\left(\left\{T_{\theta}<\infty\right\} \cap \mathcal{O}\right) \\ x_{0}, & \text { on }\left\{T_{\theta}<\infty\right\} \cap \mathcal{O}^{c}\end{cases}
$$

Clearly, $V$ is a measurable map from $\Omega$ into $\Omega$. Furthermore, Lemma 4.1 implies $P$-a.s. $V=Y$. For $n \in \mathbb{N}$ set $\gamma_{n} \triangleq T_{\theta_{n} \wedge n}$ and note that $L_{\gamma_{n}}=\theta_{n} \wedge n$. It follows from [35, Proposition V.1.4] that for all $t \in \mathbb{R}_{+}$and $n \in \mathbb{N}$

$$
L_{t \wedge \gamma_{n}}=\int_{0}^{L_{t \wedge \gamma_{n}}} \mathfrak{f}^{-1}\left(X_{s}\right) d T_{s}=\int_{0}^{t \wedge \gamma_{n}} \mathfrak{f}^{-1}\left(X_{L_{s}}\right) d T_{L_{s}}=\int_{0}^{t \wedge \gamma_{n}} \mathfrak{f}^{-1}\left(X_{L_{s}}\right) d s
$$

In other words, we have for all $n \in \mathbb{N}$

$$
\mathbb{I}_{\left\{t \leq \gamma_{n}\right\}} d L_{t}=\mathbb{I}_{\left\{t \leq \gamma_{n}\right\}} \mathfrak{f}^{-1}\left(X_{L_{t}}\right) d t
$$

Using (4.7) and again [35, Proposition V.1.4], we obtain for every locally bounded Borel function $\mathfrak{g}: \mathbb{R}^{d} \rightarrow \mathbb{R}$ that for all $t \in \mathbb{R}_{+}$and $n \in \mathbb{N}$

$$
\int_{0}^{t \wedge \gamma_{n}} \frac{\mathfrak{g}\left(Y_{s}\right) d s}{\mathfrak{f}\left(Y_{s}\right)}=\int_{0}^{t \wedge \gamma_{n}} \frac{\mathfrak{g}\left(X_{L_{s}}\right) d s}{\mathfrak{f}\left(X_{L_{s}}\right)}=\int_{0}^{t \wedge \gamma_{n}} \mathfrak{g}\left(X_{L_{s}}\right) d L_{s}=\int_{0}^{L_{t \wedge \gamma_{n}}} \mathfrak{g}\left(X_{s}\right) d s
$$

Note that $L_{t}$ is an $\mathbf{F}$-stopping time and define the time-changed filtration $\mathbf{G}=\left(\mathcal{G}_{t}\right)_{t \geq 0} \triangleq$ $\left(\mathcal{F}_{L_{t}}\right)_{t \geq 0}$. Because $\left(L_{t}\right)_{t \geq 0}$ is right-continuous, also $\mathbf{G}$ is right-continuous, and, because $\theta_{n} \wedge n$ is an F-stopping time, [20, Lemma 10.5] implies that $\gamma_{n}=T_{\theta_{n} \wedge n}$ is a G-stopping time and that $t \mapsto L_{t \wedge \gamma_{n}}$ is an increasing sequence of $\mathbf{F}$-stopping times. We set

$$
\mathfrak{K} f \triangleq\langle\nabla f, \mathfrak{b}\rangle+\frac{1}{2} \operatorname{tr}\left(\nabla^{2} f \mathfrak{a}\right), \quad f \in C^{2}\left(\mathbb{R}^{d}\right) .
$$

By the definition of the MP $\left(\mathfrak{a}, \mathfrak{b}, x_{0}\right)$, the process

$$
f\left(X_{\cdot \wedge \theta_{n}}\right)-f\left(x_{0}\right)-\int_{0}^{\cdot \wedge \theta_{n}} \mathfrak{K} f\left(X_{s}\right) d s
$$

is a $P$-martingale. Recall further that $L_{t \wedge \gamma_{n}} \leq \theta_{n} \wedge n$. Using (4.8) and the optional 
stopping theorem, for $s<t, n \in \mathbb{N}$ and $f \in C^{2}\left(\mathbb{R}^{d}\right)$ we obtain that $P$-a.s.

$$
\begin{aligned}
E^{P}\left[f\left(Y_{t \wedge \gamma_{n}}\right)\right. & \left.-f\left(x_{0}\right)-\int_{0}^{t \wedge \gamma_{n}} \frac{\mathfrak{K} f\left(Y_{r}\right) d r}{\mathfrak{f}\left(V_{r}\right)} \mid \mathcal{G}_{s}\right] \\
& =E^{P}\left[f\left(X_{L_{t \wedge \gamma_{n}} \wedge \theta_{n} \wedge n}\right)-f\left(x_{0}\right)-\int_{0}^{L_{t \wedge \gamma_{n}} \wedge \theta_{n} \wedge n} \mathfrak{K} f\left(X_{r}\right) d r \mid \mathcal{F}_{L_{s}}\right] \\
& =f\left(X_{L_{t \wedge \gamma_{n}} \wedge \theta_{n} \wedge n \wedge L_{s}}\right)-f\left(x_{0}\right)-\int_{0}^{L_{t \wedge \gamma_{n}} \wedge \theta_{n} \wedge n \wedge L_{s}} \mathfrak{K} f\left(X_{r}\right) d r \\
& =f\left(X_{L_{s \wedge \gamma_{n}}}\right)-f\left(x_{0}\right)-\int_{0}^{L_{s \wedge \gamma_{n}}} \mathfrak{K} f\left(X_{r}\right) d r \\
& =f\left(Y_{s \wedge \gamma_{n}}\right)-f\left(x_{0}\right)-\int_{0}^{s \wedge \gamma_{n}} \frac{\mathfrak{K} f\left(Y_{r}\right) d r}{\mathfrak{f}\left(Y_{r}\right)} .
\end{aligned}
$$

This yields that

$$
f\left(V \cdot \wedge \gamma_{n}\right)-f\left(x_{0}\right)-\int_{0}^{\cdot \wedge \gamma_{n}} \frac{\mathfrak{K} f\left(V_{r}\right) d r}{\mathfrak{f}\left(V_{r}\right)}
$$

is a $P$-martingale for the $P$-augmentation of $\mathbf{G}$, which we denote by $\mathbf{G}^{P}$. Note that $\theta_{n}(V)$ is a $\mathbf{G}^{P}$-stopping time. Recalling that $P$-a.s. $\gamma_{n} \nearrow T_{\theta}=\theta(V)$ and $t \wedge \theta_{n}(V)<\theta(V)$, the dominated convergence theorem yields for all $s<t, n \in \mathbb{N}$ and $f \in C^{2}\left(\mathbb{R}^{d}\right)$ that $P$-a.s.

$$
\begin{aligned}
E^{P}\left[f\left(V_{t \wedge \theta_{n}(V)}\right)\right. & \left.-f\left(x_{0}\right)-\int_{0}^{t \wedge \theta_{n}(V)} \frac{\mathfrak{K} f\left(V_{r}\right) d r}{\mathfrak{f}\left(V_{r}\right)} \mid \mathcal{G}_{s}^{P}\right] \\
& =\lim _{m \rightarrow \infty} E^{P}\left[f\left(Y_{t \wedge \theta_{n}(V) \wedge \gamma_{m}}\right)-f\left(x_{0}\right)-\int_{0}^{t \wedge \theta_{n}(V) \wedge \gamma_{m}} \frac{\mathfrak{K} f\left(V_{r}\right) d r}{\mathfrak{f}\left(V_{r}\right)} \mid \mathcal{G}_{s}^{P}\right] \\
& =\lim _{m \rightarrow \infty}\left(f\left(V_{s \wedge \theta_{n}(V) \wedge \gamma_{m}}\right)-f\left(x_{0}\right)-\int_{0}^{s \wedge \theta_{n}(V) \wedge \gamma_{m}} \frac{\mathfrak{K} f\left(V_{r}\right) d r}{\mathfrak{f}\left(V_{r}\right)}\right) \\
& =f\left(V_{s \wedge \theta_{n}(V)}\right)-f\left(x_{0}\right)-\int_{0}^{s \wedge \theta_{n}(V)} \frac{\mathfrak{K} f\left(V_{r}\right) d r}{\mathfrak{f}\left(V_{r}\right)} .
\end{aligned}
$$

Using the tower rule, we conclude that

$$
f\left(V_{\cdot \wedge \theta_{n}(V)}\right)-f\left(x_{0}\right)-\int_{0}^{\cdot \wedge \theta_{n}(V)} \frac{\mathfrak{K} f\left(V_{r}\right) d r}{\mathfrak{f}\left(V_{r}\right)}
$$

is a $P$-martingale for the filtration generated by $V$. Consequently, the push-forward $P \circ V^{-1}$ solves the MP $\left(\mathfrak{f}^{-1} \mathfrak{a}, \mathfrak{f}^{-1} \mathfrak{b}, x_{0}\right)$, which is part (i) of Theorem 2.2. Recalling (4.1) shows the formula (2.1), i.e., part (ii) of Theorem 2.2.

To prove the uniqueness claim in Theorem 2.2, we introduce a right-continuous measurable process $U$. We define

$$
S_{t} \triangleq \int_{0}^{t \wedge \theta} \mathfrak{f}^{-1}\left(X_{s}\right) d s, \quad A_{t} \triangleq \inf \left(s \in \mathbb{R}_{+}: S_{s}>t\right), \quad t \in \mathbb{R}_{+}
$$

and

$$
U_{t} \triangleq \begin{cases}X_{A_{t}}, & t<S_{\theta} \\ \Delta, & t \geq S_{\theta}\end{cases}
$$

Using (4.1) and (4.7), we obtain $P$-a.s. for all $t \in \mathbb{R}_{+}$

$$
S_{t} \circ V=\int_{0}^{t \wedge T_{\theta}} \mathfrak{f}^{-1}\left(Y_{s}\right) d s=\lim _{n \rightarrow \infty} \int_{0}^{t \wedge \gamma_{n} \wedge n} \mathfrak{f}^{-1}\left(X_{L_{s}}\right) d s=\lim _{n \rightarrow \infty} L_{t \wedge \gamma_{n} \wedge n}=L_{\left(t \wedge T_{\theta}\right)-\cdot} .
$$


In particular, $P$-a.s. $S_{\theta} \circ V=\theta$. We deduce $P$-a.s. $A_{t} \circ V=T_{t}$ for all $t<\theta$, which implies $P$-a.s. $X_{A_{t}} \circ V=X_{L_{T_{t}}}=X_{t}$ for all $t<S_{\theta} \circ V=\theta$. We conclude that $P$-a.s. $U \circ V=X$.

To prove the last claim in Theorem 2.2, suppose that $P \circ V^{-1}$ is the unique solution to the MP $\left(\mathfrak{f}^{-1} \mathfrak{a}, \mathfrak{f}^{-1} \mathfrak{b}, x_{0}\right)$. For $n \in \mathbb{N}$ let $0 \leq t_{1}<t_{2}<\cdots<t_{n}<\infty$ and $G_{1}, \ldots, G_{n} \in \mathcal{B}\left(\mathbb{R}_{\Delta}^{d}\right)$. Let $Q$ be a second solution to the $\operatorname{MP}\left(\mathfrak{a}, \mathfrak{b}, x_{0}\right)$. Then, the push-forward measures $P \circ V^{-1}$ and $Q \circ V^{-1}$ both solve the $\operatorname{MP}\left(\mathfrak{f}^{-1} \mathfrak{a}, \mathfrak{f}^{-1} \mathfrak{b}, x_{0}\right)$ and we deduce from the uniqueness assumption that

$$
\begin{aligned}
P\left(X_{t_{1}} \in G_{1}, \ldots, X_{t_{n}} \in G_{n}\right) & =P \circ V^{-1}\left(U_{t_{1}} \in G_{1}, \ldots, U_{t_{n}} \in G_{n}\right) \\
& =Q \circ V^{-1}\left(U_{t_{1}} \in G_{1}, \ldots, U_{t_{n}} \in G_{n}\right) \\
& =Q\left(X_{t_{1}} \in G_{1}, \ldots, X_{t_{n}} \in G_{n}\right) .
\end{aligned}
$$

By a monotone class argument, $P=Q$. The proof is complete.

\section{A Proof of Propositions 2.6 (i) and 2.9}

In this appendix we explain that Propositions 2.6 (i) and 2.9 can be deduced from [12, Corollary 3.4]. The difference between the setting in [12] and ours is that the underlying path space in [12] is slightly bigger and allows explosion in a discontinuous manner. We now introduce the continuous version of the path space in [12]. Let $\Sigma$ be the space of right-continuous functions $\omega: \mathbb{R}_{+} \rightarrow \mathbb{R}_{\Delta}^{d}$ which are continuous on $[0, \theta(\omega))$ and $\omega(t)=\Delta$ for $t \geq \theta(\omega)=\inf \left(t \in \mathbb{R}_{+}: \omega(t)=\Delta\right)$. Let $Y$ be the coordinate process on $\Sigma$ and define $\mathcal{A} \triangleq \sigma\left(Y_{t}, t \in \mathbb{R}_{+}\right), \mathcal{A}_{t}^{o} \triangleq \sigma\left(Y_{s}, s \in[0, t]\right)$ and $\mathcal{A}_{t} \triangleq \bigcap_{s>t} \mathcal{A}_{s}^{o}$ for $t \in \mathbb{R}_{+}$. The MP $\left(\mathfrak{a}, \mathfrak{b}, x_{0}\right)$ on $\left(\Sigma, \mathcal{A}, \mathbf{A}=\left(\mathcal{A}_{t}\right)_{t \geq 0}\right)$ is defined in the same manner as on the filtered space $(\Omega, \mathcal{F}, \mathbf{F})$ with the additional requirement that a solution $P$ has to satisfy $P$-a.s. $\theta_{n}(Y)<\theta(Y)$ on $\{\theta(Y)<\infty\}$ for all $n \in \mathbb{N}$.

Equivalently, one could define the martingale problem as follows: Using the convention that all functions $f$ on $\mathbb{R}^{d}$ are extended to $\mathbb{R}_{\Delta}^{d}$ by setting $f(\Delta) \equiv 0$, we say that $P$ solves the $\operatorname{MP}\left(\mathfrak{a}, \mathfrak{b}, x_{0}\right)$ on $(\Sigma, \mathcal{A}, \mathbf{A})$ if $P\left(X_{0}=x_{0}\right)=1$ and for all $f \in C_{c}^{2}\left(\mathbb{R}^{d}\right)$ the process

$$
f\left(Y_{\cdot \wedge \theta_{n}(Y)}\right)-f\left(x_{0}\right)-\int_{0}^{\cdot \wedge \theta_{n}(Y)}\left(\left\langle\nabla f\left(Y_{s}\right), \mathfrak{b}\left(Y_{s}\right)\right\rangle+\frac{1}{2} \operatorname{tr}\left(\nabla^{2} f\left(Y_{s}\right) \mathfrak{a}\left(Y_{s}\right)\right)\right) d s
$$

is a $P$-martingale. In this case, it always holds that $P$-a.s. $\theta_{n}(Y)<\theta(Y)$ on $\{\theta(Y)<\infty\}$ for all $n \in \mathbb{N}$ and consequently, the definitions are equivalent. This follows from an argument in the proof of [7, Lemma 3.1]: Let $f_{k} \in C_{c}^{2}\left(\mathbb{R}^{d}\right)$ be such that $0 \leq f_{k} \leq 1$ and $f_{k}(x)=1$ whenever $\|x\| \leq k$. The process $f_{k}\left(Y \cdot \wedge \theta_{n}(Y)\right)-f_{k}\left(x_{0}\right)$ is a $P$-martingale for all $k>n$, because $\nabla f_{k}\left(Y_{s}\right)$ and $\nabla^{2} f_{k}\left(Y_{s}\right)$ vanish for all $s<\theta_{n}(Y)$. Thus, by dominated convergence,

$$
0=\lim _{k \rightarrow \infty} E^{P}\left[f_{k}\left(Y_{t \wedge \theta_{n}(Y)}\right)-f_{k}\left(x_{0}\right)\right]=P\left(t \wedge \theta_{n}(Y)<\theta(Y)\right)-1, \quad t \in \mathbb{R}_{+} .
$$

This shows that $P$-a.s. $\theta_{n}(Y)<\theta(Y)$ on $\{\theta(Y)<\infty\}$ for all $n \in \mathbb{N}$.

A third equivalent definition is the following: A probability measure $P$ on $(\Sigma, \mathcal{A})$ solves the MP $\left(\mathfrak{a}, \mathfrak{b}, x_{0}\right)$ if $P\left(Y_{0}=x_{0}\right)=1$ and for all $n \in \mathbb{N}$ the stopped process $Y_{\cdot \wedge \theta_{n}(Y)}$ is an $\mathbb{R}^{d}$-valued continuous semimartingale with semimartingale characteristics $\left(B^{n}, C^{n}, 0\right)$, where

$$
B^{n}=\int_{0}^{\cdot \wedge \theta_{n}(Y)} \mathfrak{b}\left(Y_{s}\right) d s, \quad C^{n}=\int_{0}^{\cdot \wedge \theta_{n}(Y)} \mathfrak{a}\left(Y_{s}\right) d s
$$

see [20, Theorem 13.55] for more details. This relates the martingale problem to the semimartingale problem defined in [12, Definition 2.1].

Lemma A.1. The path space $\Omega$ is a measurable subset of the path space $\Sigma$, i.e., $\Omega \in \mathcal{A}$. 
Proof. Let $d$ be a metric on $\mathbb{R}_{\Delta}^{d}$ (which implicitely induces the topology) and set $Z_{t} \triangleq$ $\lim \sup _{s \rightarrow t-, s \in \mathbb{Q}_{+}} d\left(Y_{s}, \Delta\right)$ for $t>0$. Due to [14, Theorem IV.17], the process $\left(Z_{t}\right)_{t>0}$ is progressively measurable for the filtration A. Moreover, [17, Lemma 2.2.8] yields that

$$
\Omega=\{\theta(Y) \notin(0, \infty)\} \cup\left\{Z_{\theta(Y)}=0, \theta(Y) \in(0, \infty)\right\} .
$$

Hence, the claim follows.

We define the following well-posedness condition:

(C) $\mathfrak{a}$ and $\mathfrak{b}$ are locally bounded and for all $x \in \mathbb{R}^{d}$ the $\operatorname{MP}(\mathfrak{a}, \mathfrak{b}, x)$ on $(\Omega, \mathcal{F}$, F $)$ has a unique solution $P_{x}$.

The following lemma relates the martingale problems on the path spaces $\Sigma$ and $\Omega$.

Lemma A.2. Suppose that (C) holds. Then, $P_{x}$, extended to $(\Sigma, \mathcal{A})$, see Lemma A.1, is also the unique solution to the $\operatorname{MP}(\mathfrak{a}, \mathfrak{b}, x)$ on $(\Sigma, \mathcal{A}, \mathbf{A})$.

Proof. On an intuitive level, any solution to the $\operatorname{MP}(\mathfrak{a}, \mathfrak{b}, x)$ on $(\Sigma, \mathcal{A}, \mathbf{A})$ should coincide locally with $P_{x}$ and consequently, explosion should happen in the same manner for both problems. We now make this intuition precise.

The following local uniqueness property of well-posed martingale problems can be proven similar to [11, Lemma 9.1], cf. [40, Exercise 11.5.1] and [17, Theorem 4.6.1].

Lemma A.3. Suppose that $(\mathrm{C})$ holds, let $\tau$ be an $\left(\mathcal{F}^{o}\right)_{t \geq 0}$-stopping time and let $R$ be a probability measure on $(\Omega, \mathcal{F})$ with $R\left(X_{0}=x\right)=1$ and with the property that for all $n \in \mathbb{N}$ and $f \in C^{2}\left(\mathbb{R}^{d}\right)$ the process

$$
f\left(X_{\cdot \wedge \tau \wedge \theta_{n}}\right)-f(x)-\int_{0}^{\cdot \wedge \tau \wedge \theta_{n}}\left(\left\langle\nabla f\left(X_{s}\right), \mathfrak{b}\left(X_{s}\right)\right\rangle+\frac{1}{2} \operatorname{tr}\left(\nabla^{2} f\left(X_{s}\right) \mathfrak{a}\left(X_{s}\right)\right)\right) d s
$$

is an $R$-martingale. Then, $R=P_{x}$ on $\mathcal{F}_{\tau}^{o}$.

Note that $\theta_{n}$ and $\theta_{n}(Y)$ are stopping times for the filtrations $\left(\mathcal{F}_{t}^{o}\right)_{t \geq 0}$ and $\left(\mathcal{A}_{t}^{o}\right)_{t \geq 0}$, respectively. In the first case this is a well-known fact ([35, Proposition I.4.5]), because the coordinate process on $\Omega$ has continuous paths. On $\Sigma$ the coordinate process is not continuous and the classical result does not apply, but its proof can be adapted easily:

$$
\left\{\theta_{n}(Y) \leq t\right\}=\left\{\inf _{q \in \mathbb{Q} \cap[0, t]} d\left(Y_{q}, B_{n}^{c}\right)=0, Y_{t} \neq \Delta\right\} \cup\left\{Y_{t}=\Delta\right\} \in \mathcal{A}_{t}^{o}, \quad t \in \mathbb{R}_{+},
$$

where $d$ is a metric on $\mathbb{R}_{\Delta}^{d}$ and $B_{n}^{c} \triangleq\left\{x \in \mathbb{R}^{d}:\|x\| \geq n\right\} \cup\{\Delta\}$.

Let $Q$ be a solution to the $\operatorname{MP}(\mathfrak{a}, \mathfrak{b}, x)$ on $(\Sigma, \mathcal{A}, \mathbf{A})$. By definition of the martingale problem, the push-forward $R \equiv Q \circ Y_{\cdot \wedge \theta_{n}(Y)}^{-1}$ satisfies the assumptions in Lemma A.3 for $\tau \equiv \theta_{n}$. Thus, Lemma A.3 implies that $R=P_{x}$ on $\mathcal{F}_{\theta_{n}}^{o}$. Provided $P_{x}$ is extended to $(\Sigma, \mathcal{A})$, this implies that $Q=P_{x}$ on $\mathcal{A}_{\theta_{n}(Y)}^{o}$. Now, because $\bigvee_{n \in \mathbb{N}} \mathcal{A}_{\theta_{n}(Y)-}^{o}=\mathcal{A}_{\theta(Y)-}^{o}$, a monotone class argument shows that $Q=P_{x}$ on $\mathcal{A}_{\theta(Y)-}^{o}$. Finally, note that $\mathcal{A}_{\theta(Y)-}^{o}=\mathcal{A}$, which follows from the observation

$$
\left\{Y_{t} \in G\right\}= \begin{cases}\{\theta(Y) \leq t\} \cup\left(\left\{Y_{t} \in G\right\} \cap\{\theta(Y)>t\}\right), & \Delta \in G, \\ \left\{Y_{t} \in G\right\} \cap\{\theta(Y)>t\}, & \Delta \notin G,\end{cases}
$$

for all $t \in \mathbb{R}_{+}$and $G \in \mathcal{B}\left(\mathbb{R}_{\Delta}^{d}\right)$. The proof is complete.

We are in the position to deduce Propositions 2.6 (i) and 2.9 from [12, Corollary 3.4]. Note that [12, Standing Assumption 3.1], which is only concerned with the existence of $Q_{x_{0}}$, is implied by (S3), and that [12, Standing Assumption 3.2] and the first part of [12, Standing Assumption 3.3] are not needed in our continuous setting. Note that (S4) implies [12, Standing Assumption 3.4]. Thus, Propositions 2.6 (i) and 2.9 follow from [12, Corollary 3.4], because its uniqueness assumption is implied by Lemma A.2. 
Remark A.4. Using Lemma A.3, Propositions 2.6 (i) and 2.9 could have been proven directly without [12, Corollary 3.4]. We think it is interesting to relate the setting in [12] to ours.

\section{B Martingale problems and stochastic differential equations}

In this appendix we recall the relation of martingale problems and weak solutions of stochastic differential equations. The following definition can be viewed as a multidimensional version of [22, Definition 5.5.1].

Definition B.1. Let $\mathfrak{b}: \mathbb{R}^{d} \rightarrow \mathbb{R}^{d}$ and $\mathfrak{s}: \mathbb{R}^{d} \rightarrow \mathbb{R}^{d \times r}$ be locally bounded Borel functions and denote the adjoint of $\mathfrak{s}(x)$ by $\mathfrak{s}^{*}(x)$. We call a triplet $((\Sigma, \mathcal{A}, \mathbf{A}, P), Y, W)$ a weak solution to the $\operatorname{SDE}\left(\mathfrak{s}, \mathfrak{b}, x_{0}\right)$, if the following hold:

(i) The triplet $(\Sigma, \mathcal{A}, P)$ is a complete probability space, $\mathrm{A}$ is an augmented filtration on $(\Sigma, \mathcal{A}, P)$, and $Y=\left(Y_{t}\right)_{t \geq 0}$ and $W=\left(W_{t}\right)_{t \geq 0}$ are measurable processes on $(\Sigma, \mathcal{A})$.

(ii) The process $Y$ is $\mathbb{R}_{\Delta}^{d}$-valued, continuous and $\mathbf{A}$-adapted, and $W$ is an $r$-dimensional standard A-Brownian motion.

(iii) For every $n \in \mathbb{N}$ the process $Y$ satisfies $P$-a.s.

$$
Y_{\cdot \wedge \theta_{n}(Y)}=x_{0}+\int_{0}^{\cdot \wedge \theta_{n}(Y)} \mathfrak{b}\left(Y_{s}\right) d s+\int_{0}^{\cdot \wedge \theta_{n}(Y)} \mathfrak{s}\left(Y_{s}\right) d W_{s},
$$

where the integrals are well-defined due the local boundedness of $\mathfrak{b}$ and $\mathfrak{s}$. Moreover, we stipulate that $Y_{t}=Y_{\theta(Y)}$ for all $t \geq \theta(Y)$.

The following is a version of [17, Corollary 5.3.4] or [22, Corollary 5.4.8] for possibly explosive MPs and SDEs, see [19, Theorem IV.6.1] for a statement in a non-conservative setting with continuous coefficients. The proof is identical to the non-explosive case and omitted.

Proposition B.2. Suppose that $\mathfrak{b}: \mathbb{R}^{d} \rightarrow \mathbb{R}^{d}$ and $\mathfrak{s}: \mathbb{R}^{d} \rightarrow \mathbb{R}^{d \times r}$ are locally bounded Borel functions.

(i) If $((\Sigma, \mathcal{A}, \mathbf{A}, P), Y, W)$ is a weak solution to the $S D E\left(\mathfrak{s}, \mathfrak{b}, x_{0}\right)$, then the push-forward $P \circ Y^{-1}$ solves the $M P\left(\mathfrak{s s}^{*}, \mathfrak{b}, x_{0}\right)$.

(ii) If $Q$ solves the $M P\left(\mathfrak{s s}^{*}, \mathfrak{b}, x_{0}\right)$, then there exists a weak solution $((\Sigma, \mathcal{A}, \mathbf{A}, P), Y, W)$ to the $\operatorname{SDE}\left(\mathfrak{s}, \mathfrak{b}, x_{0}\right)$ and $Q=P \circ Y^{-1}$.

Remark B.3. In case one starts with a coefficient $\mathfrak{a}: \mathbb{R}^{d} \rightarrow \mathbb{S}^{d}$ it is always possible to find a decomposition $\mathfrak{a}=\mathfrak{s s}=\mathfrak{s} \mathfrak{s}^{*}$, where $\mathfrak{s}: \mathbb{R}^{d} \rightarrow \mathbb{S}^{d}$ is Borel, locally bounded or continuous whenever $\mathfrak{a}$ is Borel, locally bounded or continuous, respectively. Let us explain this in more detail: It is well-known that for a matrix $A \in \mathbb{S}^{d}$ there exists a unique matrix $A^{\frac{1}{2}} \in \mathbb{S}^{d}$ such that $A=A^{\frac{1}{2}} A^{\frac{1}{2}}$. Moreover, the map $S: \mathbb{S}^{d} \rightarrow \mathbb{S}^{d}$ defined by $S(A)=A^{\frac{1}{2}}$ is continuous (for the matrix-norm topology on $\mathbb{S}^{d}$ ). In fact, $S$ is even Hölder continuous with exponent $\frac{1}{2}$, which follows from the Powers-Størmer inequality ([34, Lemma 4.1]):

$$
\left\|A^{\frac{1}{2}}-B^{\frac{1}{2}}\right\|_{2} \leq \sqrt{\|A-B\|_{1}} \leq d^{\frac{1}{4}} \sqrt{\|A-B\|_{2}}, \quad A, B \in \mathbb{S}^{d},
$$

where $\|\cdot\|_{1}$ denotes the trace norm and $\|\cdot\|_{2}$ denotes the Hilbert-Schmidt norm. The function $\mathfrak{s} \equiv S(\mathfrak{a})$ has the claimed properties. Although continuity transfers from $\mathfrak{a}$ to its root $\mathfrak{s}=S(\mathfrak{a})$, the same is not necessarily true for higher regularities, see [18, Section 6.1] or [40, Section 5.2] for comments. 


\section{A few existence and uniqueness results}

In this appendix we collect some existence and uniqueness results for martingale problems. We assume that $\mathfrak{b}: \mathbb{R}^{d} \rightarrow \mathbb{R}^{d}$ and $\mathfrak{a}: \mathbb{R}^{d} \rightarrow \mathbb{S}^{d}$ are locally bounded Borel functions and we formulate the following conditions:

(A1) $\mathfrak{b}$ and $\mathfrak{a}$ are continuous.

(A2) $\left\{x \in \mathbb{R}^{d}: \int_{B_{r}(x)} \frac{d y}{\operatorname{det}(\mathfrak{a}(y))}=\infty\right.$ for all $\left.r>0\right\} \subseteq\left\{x \in \mathbb{R}^{d}: \mathfrak{b}(x)=0, \mathfrak{a}(x)=0\right\}$, where $B_{r}(x)=\left\{y \in \mathbb{R}^{d}:\|x-y\|<r\right\}$ denotes the open ball with center $x$ and radius $r$.

(A3) $\mathfrak{a}$ is continuous and $\langle\xi, \mathfrak{a}(x) \xi\rangle>0$ for all $x \in \mathbb{R}^{d}$ and $\xi \in \mathbb{R}^{d} \backslash\{0\}$.

(A4) $\mathfrak{b}$ is locally Lipschitz continuous and $\mathfrak{a}$ has a decomposition $\mathfrak{a}=\mathfrak{s \mathfrak { s } ^ { * }}$, where $\mathfrak{s}: \mathbb{R}^{d} \rightarrow$ $\mathbb{R}^{d \times r}$ is locally Lipschitz continuous.

We use this opportunity and illustrate that Theorem 2.2 can be used to prove conditions for existence and uniqueness of martingale problems.

Proposition C.1. Let $x_{0} \in \mathbb{R}^{d}$. If (A1) or (A2) holds, then there exists a solution to the $M P\left(\mathfrak{a}, \mathfrak{b}, x_{0}\right)$. If (A3) or (A4) holds, then there exists a unique solution to the $M P\left(\mathfrak{a}, \mathfrak{b}, x_{0}\right)$.

Proof. The following strategy is borrowed from the proof of [19, Theorem IV.2.3]. Let $\mathfrak{f}: \mathbb{R}^{d} \rightarrow(0, \infty)$ be a continuous function such that $\mathfrak{f a}$ and $\mathfrak{f} \mathfrak{b}$ are bounded. Such a function can be constructed as follows: Set

$$
\mathfrak{g} \triangleq \sum_{k=1}^{\infty} a_{k}^{-1} \mathbb{I}_{[k-1, k)}, \text { where } a_{k} \triangleq \sup _{\|x\| \leq k}\|\mathfrak{b}(x)\| \vee \sup _{\|x\| \leq k}\|\mathfrak{a}(x)\| \vee 1,
$$

and let $\mathfrak{z}: \mathbb{R}_{+} \rightarrow(0, \infty)$ be a continuous function $\mathfrak{z} \leq \mathfrak{g}$. Then, $\mathfrak{f}(x) \triangleq \mathfrak{z}(\|x\|)$ has the claimed properties. In case one of (A1) - (A3) holds, the MP $\left(\mathfrak{f a}, \mathfrak{f} \mathfrak{b}, x_{0}\right)$ has a (conservative) solution and in case (A3) holds the solution is even unique. With these observations at hand, Theorem 2.2 implies that existence holds for the MP $\left(\mathfrak{a}, \mathfrak{b}, x_{0}\right)$ under either of (A1) - (A4) and that uniqueness holds under (A3). That uniqueness also holds under (A4) is well-known, see [19, Theorem IV.3.1]. Finally, we provide references for the existence and uniqueness statements concerning the MP $\left(\mathfrak{f} \mathfrak{a}, \mathfrak{f} \mathfrak{b}, x_{0}\right)$ : For existence under (A1) see [40, Theorem 6.1.7], and for existence and uniqueness under (A3) see [40, Theorem 7.2.1]. Recalling Proposition B.2 and Remark B.3, existence under (A2) is implied by [36, Theorem 2].

Remark C.2. Existence under (A1) is also implied by [19, Theorem IV.2.3] and existence and uniqueness under (A3) is implied by [33, Theorem 1.13.1]. Under (A2), existence of a solution with not necessarily continuous paths (more precisely with paths in $\Sigma$ as defined in Appendix A) is implied by [25, Theorem 4.4].

Finally, we recall that Girsanov's theorem is helpful in the study of uniqueness, see also [22, Proposition 5.3.10] and [21, Proposition 18.12].

Proposition C.3. Let $\mathfrak{b}, \mathfrak{c}: \mathbb{R}^{d} \rightarrow \mathbb{R}^{d}$ and $\mathfrak{a}: \mathbb{R}^{d} \rightarrow \mathbb{S}^{d}$ be Borel functions such that $\mathfrak{a}, \mathfrak{b}, \mathfrak{a c}$ and $\langle\mathfrak{c}, \mathfrak{a c}\rangle$ are locally bounded. Assume that for all $x \in \mathbb{R}^{d}$ the $M P(\mathfrak{a}, \mathfrak{b}, x)$ has a unique solution $P_{x}$. Then, for every $x \in \mathbb{R}^{d}$ the $M P(\mathfrak{a}, \mathfrak{b}+\mathfrak{a c}, x)$ has at most one solution.

Proof. Lemma A.3, Proposition B.2 and [21, Proposition 18.12] yield that all solutions to the MP $(\mathfrak{a}, \mathfrak{b}+\mathfrak{a} \mathfrak{c}, x)$ coincide on $\mathcal{F}_{\theta_{n}}^{o}$ for all $n \in \mathbb{N}$. By a monotone class argument, this implies the claim. 
Absolute continuity/singularity of multidimensional diffusions

\section{References}

[1] C. D. Aliprantis and K. C. Border. Infinite Dimensional Analysis: A Hitchhiker's Guide. Springer Berlin Heidelberg, 3rd edition, 2006. MR-2378491

[2] C. J. K. Batty. Asymptotic stability of Schrödinger semigroups: path integral methods. Mathematische Annalen, 292(3):457-492, 1992. MR-1152946

[3] I. Ben-Ari and R. G. Pinsky. Absolute continuity/singularity and relative entropy properties for probability measures induced by diffusions on infinite time intervals. Stochastic Processes and their Applications, 115(2):179-206, 2005. MR-2111192

[4] R. N. Bhattacharya and S. Ramasubramanian. Recurrence and ergodicity of diffusions. Journal of Multivariate Analysis, 12(1):95-122, 1982. MR-0650932

[5] A. N. Borodin and P. Salminen. Some exponential integral functionals of BM $(\mu)$ and Bes (3). Journal of Mathematical Sciences, 133(3):1231-1248, 2006. MR-2092200

[6] B. Böttcher, R. Schilling, and J. Wang. Lévy Matters III: Lévy-Type Processes: Construction, Approximation and Sample Path Properties. Springer International Publishing, 2014. MR3156646

[7] P. Cheridito, D. Filipović, and M. Yor. Equivalent and absolutely continuous measure changes for jump-diffusion processes. The Annals of Applied Probability, 15(3):1713-1732, 2005. MR-2152242

[8] A. S. Cherny. General arbitrage pricing model: I - probability approach. In C. Donati-Martin, M. Émery, A. Rouault, C. Stricker, editors, Séminaire de Probabilités XL, pages 415-445, Springer Berlin Heidelberg, 2007. MR-2409020

[9] A. S. Cherny and M. Urusov. On the absolute continuity and singularity of measures on filtered spaces: Separating times. In From Stochastic Calculus to Mathematical Finance: The Shiryaev Festschrift, pages 125-168. Springer Berlin Heidelberg, 2006. MR-2233538

[10] D. Criens. Deterministic criteria for the absence and existence of arbitrage in multidimensional diffusion markets. International Journal of Theoretical and Applied Finance, 21(01):1850002, 2018. MR-3786710

[11] D. Criens. No arbitrage in continuous financial markets. Mathematics and Financial Economics, 14:461-506, 2020. MR-4098848

[12] D. Criens and K. Glau. Absolute continuity of semimartingales. Electronic Journal of Probability, 23(125):1-28, 2018. MR-3896862

[13] Z. Cui. A new proof of an Engelbert-Schmidt type zero-one law for time-homogeneous diffusions. Statistics \& Probability Letters, 89:118-123, 2014. MR-3191469

[14] C. Dellacherie and P.-A. Meyer. Probabilities and Potential. North-Holland, 1978. MR-0521810

[15] F. Delbaen and W. Schachermayer. The Mathematics of Arbitrage. Springer Berlin Heidelberg, 2006. MR-2200584

[16] H.-J. Engelbert and W. Schmidt. On the behaviour of certain Bessel functional. An application to a class of stochastic differential equations. Mathematische Nachrichten, 131(1):219-234, 1987. MR-0908813

[17] S. N. Ethier and T. G. Kurtz. Markov Processes: Characterization and Convergence. Wiley, 2005. MR-0838085

[18] A. Friedman. Stochastic Differential Equations and Applications: Volume 1. Academic Press, 1975. MR-0494490

[19] N. Ikeda and S. Watanabe. Stochastic Differential Equations and Diffusion Processes. Elsevier Science, 1989. MR-1011252

[20] J. Jacod. Calcul stochastique et problèmes de martingales. Springer Berlin Heidelberg New York, 1979. MR-0542115

[21] O. Kallenberg. Foundations of Modern Probability. Probability and Its Applications. Springer New York, 1997. MR-1464694

[22] I. Karatzas and S. E. Shreve. Brownian Motion and Stochastic Calculus. Springer New York, 2nd edition, 1991. MR-1121940 
[23] D. Khoshnevisan, P. Salminen, and M. Yor. A note on a.s. finiteness of perpetual integral functionals of diffusions. Electronic Communications in Probability, 11:108-117, 2006. MR2231738

[24] F. Kühn. Perpetual integrals via random time changes. Bernoulli, 25(3):1755-1769, 2019. MR-3961229

[25] V. P. Kurenok and A. N. Lepeyev. On multi-dimensional SDEs with locally integrable coefficients. The Rocky Mountain Journal of Mathematics, 38(1):139-174, 2008. MR-2397030

[26] A. Lyasoff. The two fundamental theorems of asset pricing for a class of continuous-time financial markets. Mathematical Finance, 24(3):485-504, 2014. MR-3274938

[27] H. P. McKean. Stochastic Integrals. Academic Press, 1969. MR-0247684

[28] A. Mijatović and M. Urusov. Convergence of integral functionals of one-dimensional diffusions. Electronic Communications in Probability, 17(61):1-13, 2012. MR-3005734

[29] A. Mijatović and M. Urusov. On the martingale property of certain local martingales. Probability Theory and Related Fields, 152:1-30, 2012. MR-2875751

[30] A. Mijatović and M. Urusov. Deterministic criteria for the absence of arbitrage in onedimensional diffusion models. Finance and Stochastics, 16(2):225-247, 2012. MR-2903623

[31] P. Mörters and Y. Peres. Brownian Motion. Cambridge Series in Statistical and Probabilistic Mathematics. Cambridge University Press, 2010. MR-2604525

[32] M. Musiela. On Kac functionals of one-dimensional diffusions. Stochastic Processes and their Applications, 22(1):79-88, 1986. MR-0852384

[33] R. G. Pinsky. Positive Harmonic Functions and Diffusion. Cambridge University Press, 1995. MR-1326606

[34] R. T. Powers and E. Størmer. Free states of the canonical anticommutation relations. Communications in Mathematical Physics, 16(1):1-33, 1970. MR-0269230

[35] D. Revuz and M. Yor. Continuous Martingales and Brownian Motion. Springer Berlin Heidelberg, 3rd edition, 1999. MR-1725357

[36] A. Rozkosz and L. Słomiński. On existence and stability of weak solutions of multidimensional stochastic differential equations with measurable coefficients. Stochastic Processes and their Applications, 37(2):187-197, 1991. MR-1102869

[37] J. Ruf. The martingale property in the context of stochastic differential equations. Electronic Communications in Probability, 20(34):1-10, 2015. MR-3342168

[38] A. N. Shiryaev. Optimal Stopping Rules. Springer Berlin Heidelberg, 1978. MR-0468067

[39] C. Sin. Complications with stochastic volatility models. Advances in Applied Probability, 30(1):256-268, 1998. MR-1618849

[40] D. W. Stroock and S. R. S. Varadhan. Multidimensional Diffussion Processes. Springer Berlin Heidelberg, 1997. MR-0532498

[41] V. A. Volkonskii. Random substitution of time in strong Markov processes. Theory of Probability \& Its Applications, 3(3):310-326, 1958. MR-0100919

[42] X.-X. Xue. A zero-one law for integral functionals of the Bessel process. In Jacques Azéma, Marc Yor, and Paul André Meyer, editors, Séminaire de Probabilités XXIV 1988/89, pages 137-153, Springer Berlin Heidelberg, 1990. MR-1071537

Acknowledgments. The author thanks the anonymous referee for many helpful comments. 